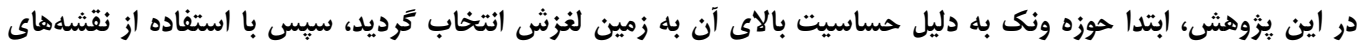

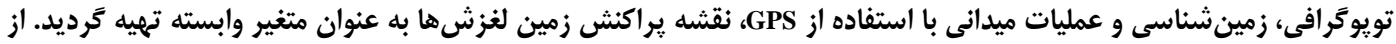

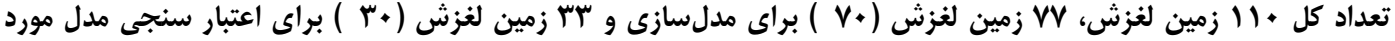

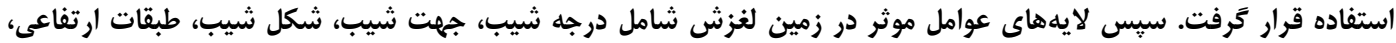

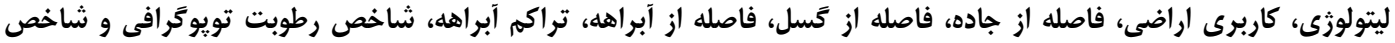

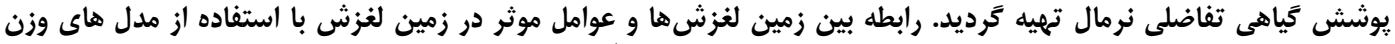

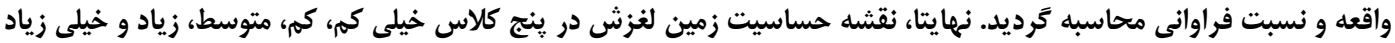

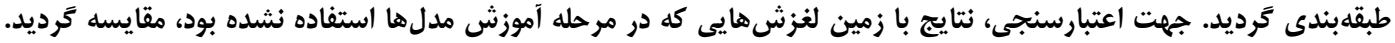

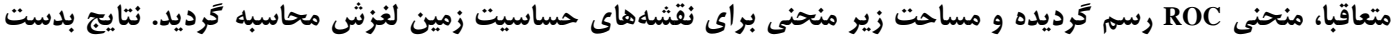

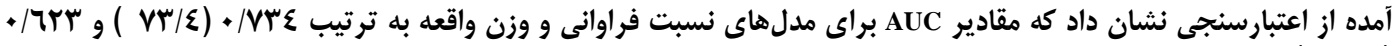

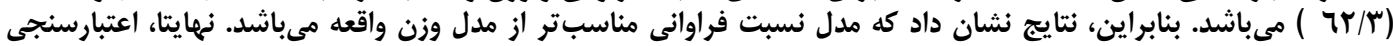

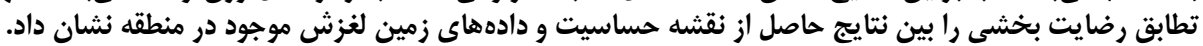

وازههاى كليدى: بهينهبندى، زمين لغزش، روش نسبت فراوانى، روش وزن واقعه، حوزه ونك

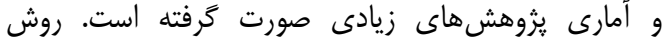

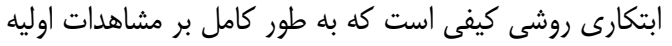

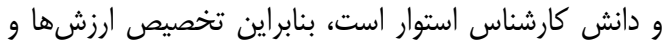

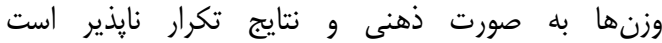

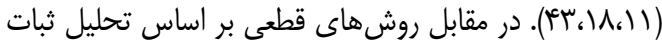

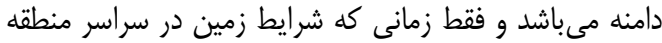

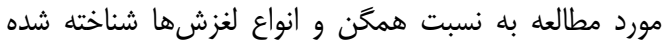

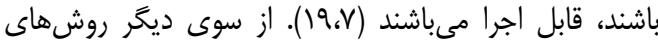

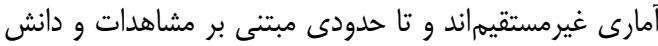

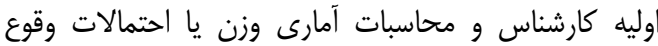

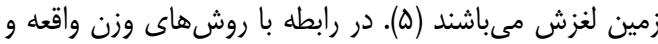

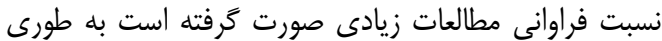

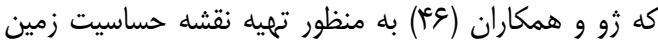

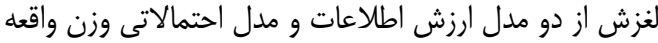

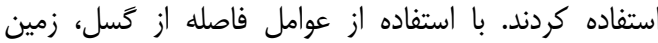

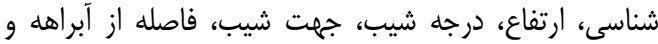

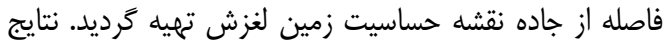

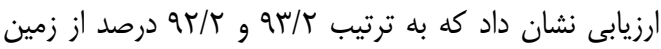

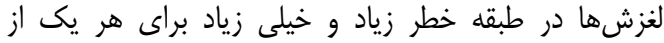

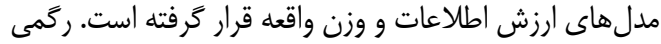

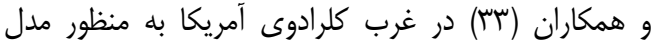

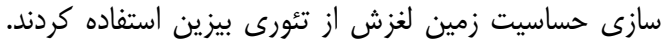

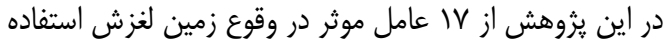

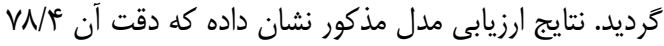

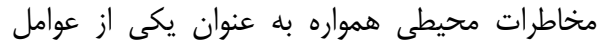

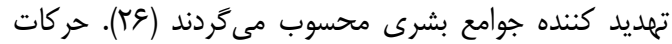

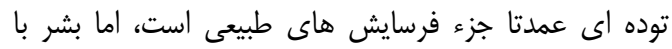

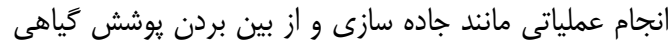

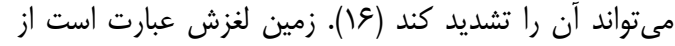

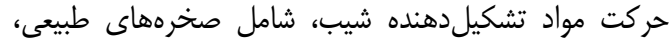

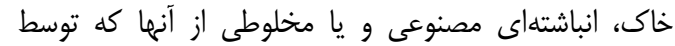

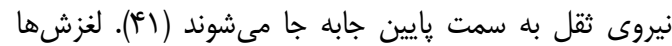

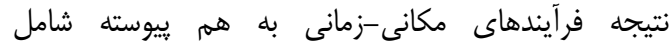
فرايندهاى هيدرولوزيك (بارش، تبخير و وآبهاني آنهاى زير زمينى)،

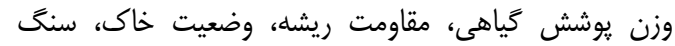

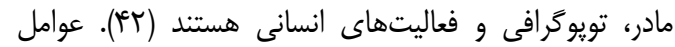

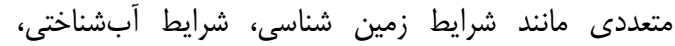

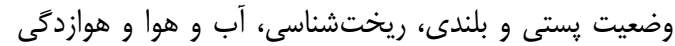

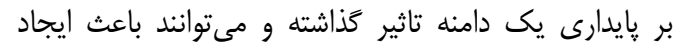

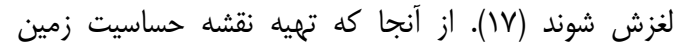

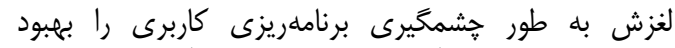

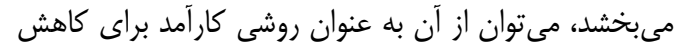

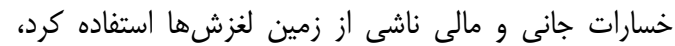

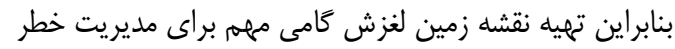

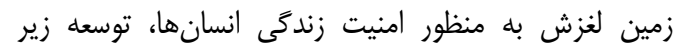

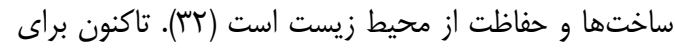

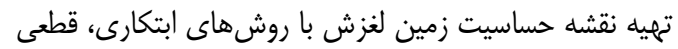




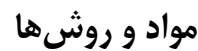 منطقه مورد مطالعه}

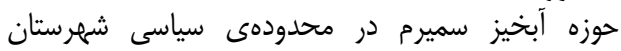

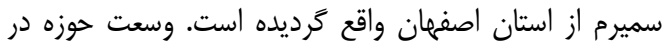

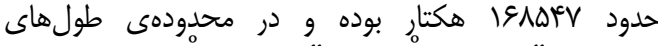

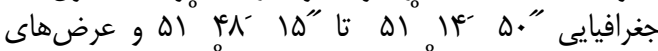

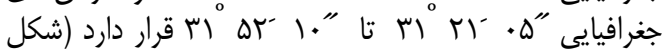

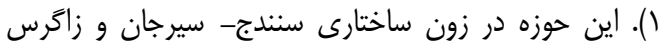

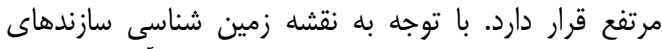

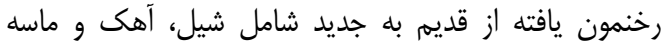

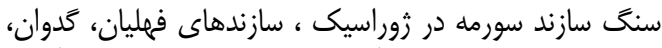

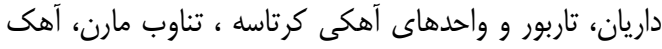

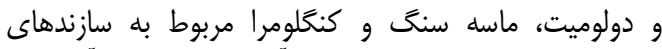

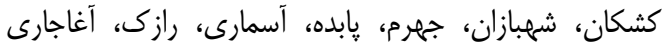

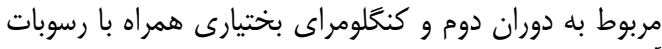

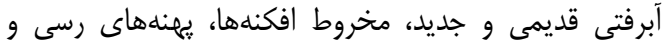

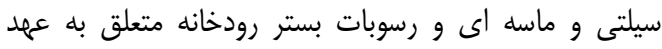

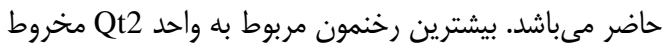

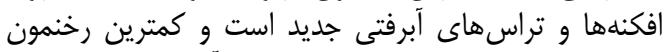

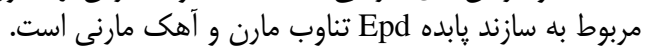

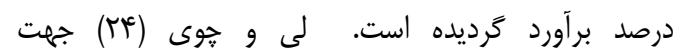
يرينهبندى حساسيت زمين لغزش از

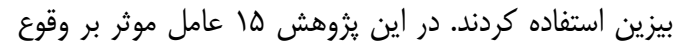

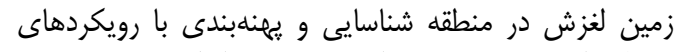

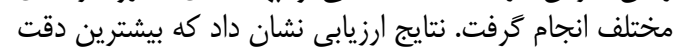

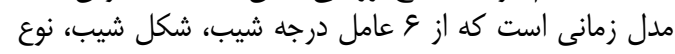

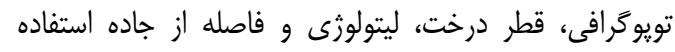

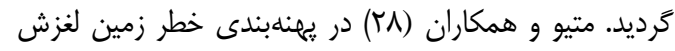

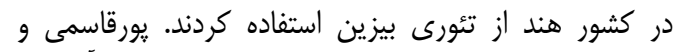

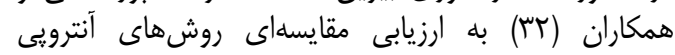

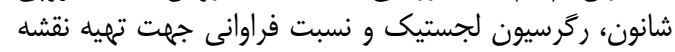

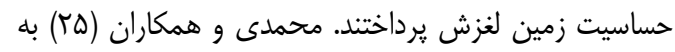

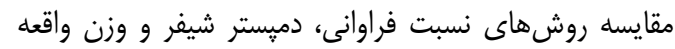

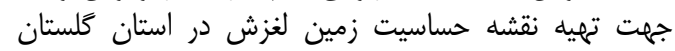

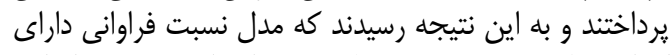

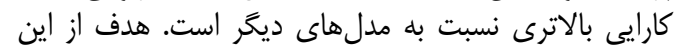

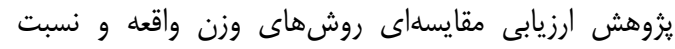

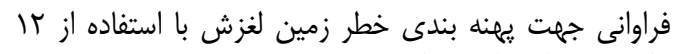

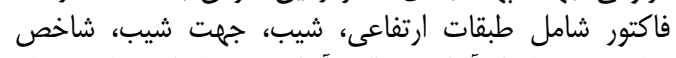

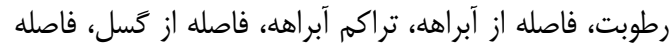

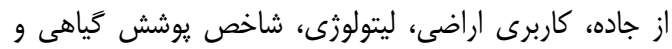
شكل شيب مىباشد.

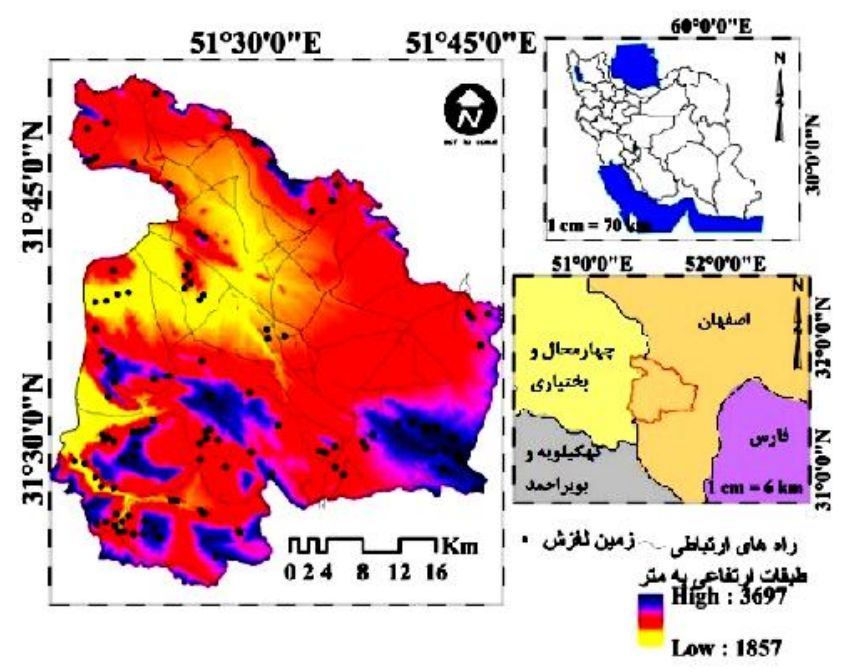

شكل 1- موقعيت منطقه مورد مطالعه

Figure 1. Location of the study area

ارتفاعى، شيب، جهت شيب، شاخص رطوبت، فاصله از آبراهه،

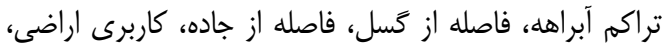

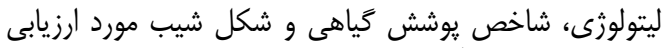

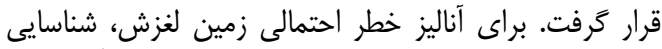

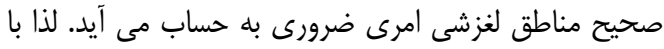

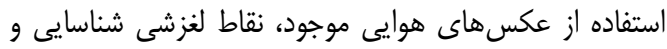

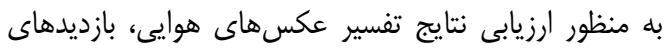

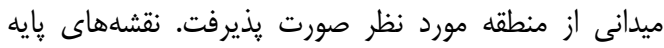
مورد استفاده در اين يزوهش عبار مورد نظد از نقشه زمين شناسى در
روش تحقيق شناسايى عوامل موثر در وقوع زمين لغزش، مهرمترين

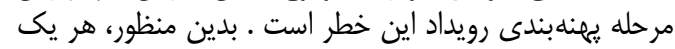

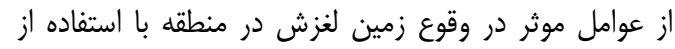

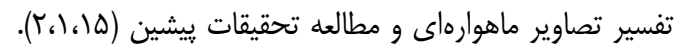

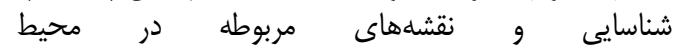

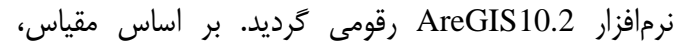

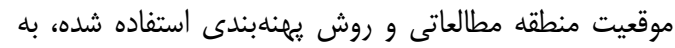

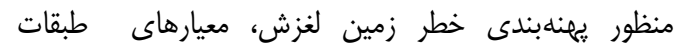


(•رد). جهت توليد شكل شيب از مدل رقومى ارتفاعى استفاده

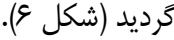

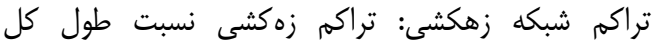

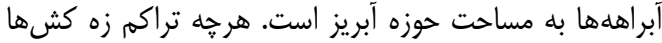

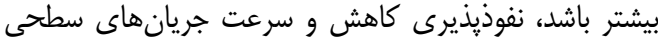

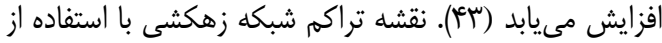

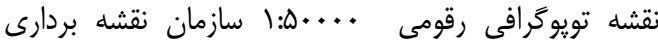

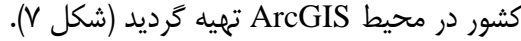

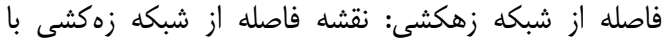

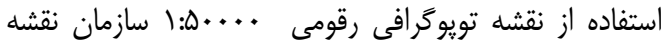

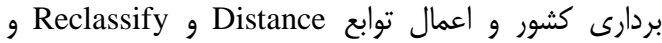
كردن آن با نقشه براكنش زمين لغز لنشها در محيط ArcGIS

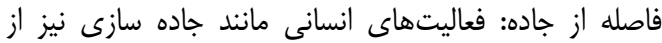

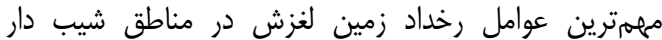

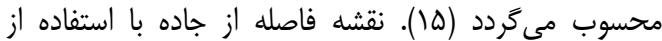

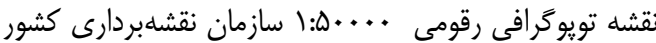

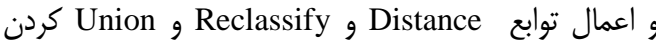
آن با نقشه يراكنش زمين لغزشها دراب در محيط ArcGIS تهيه

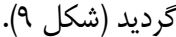

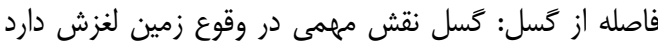

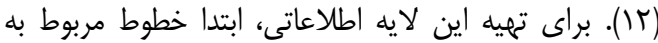

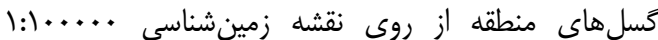

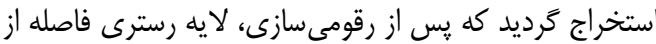
كسل در ه كلاس در محيط AreGIS تعييه كرديد (شكل

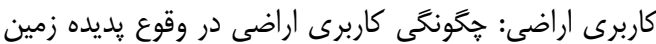

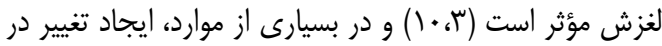

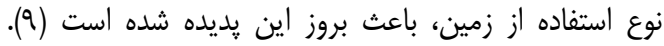

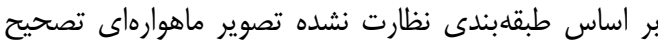

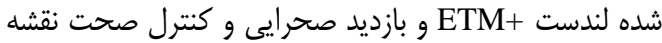

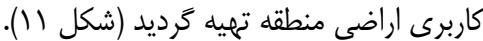

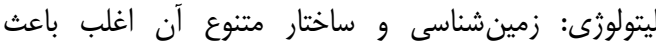

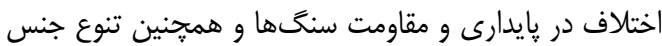

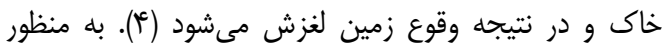

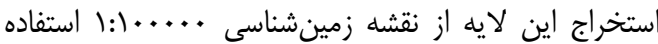

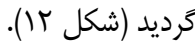

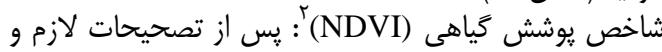

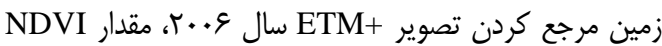

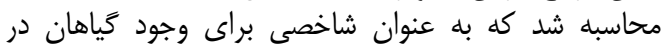
آبراهdها از آن استفاده شد (•r) (شكل سار).

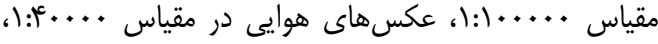

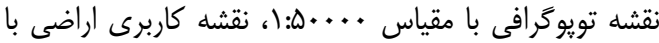

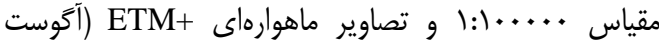

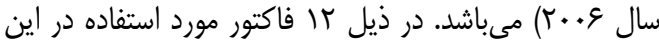

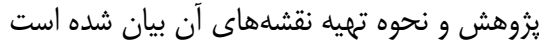

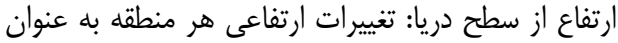

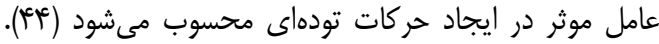

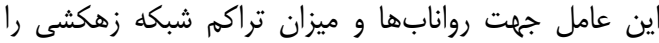

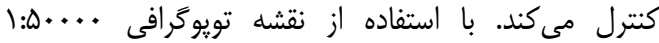

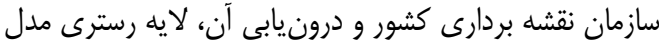

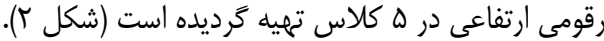

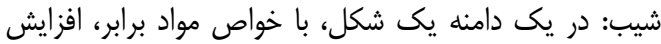

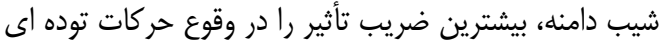

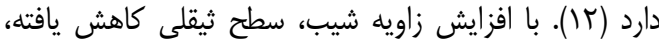

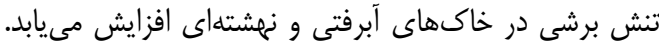

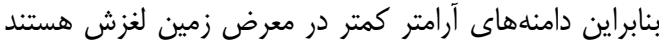

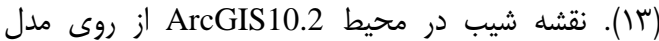

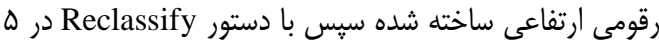

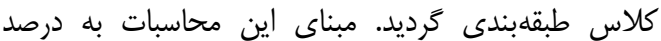
مىباشد (شكل بَ). جهت شيب: جهت شيب نشاندهن بنده تأثير متفاوت نور آفتاب،

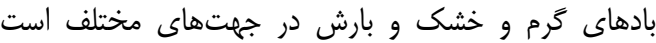

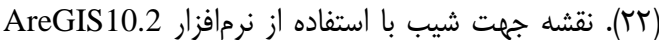

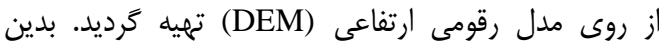

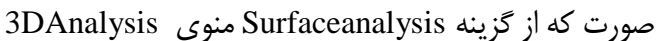

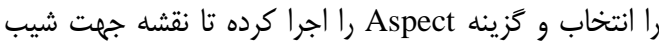

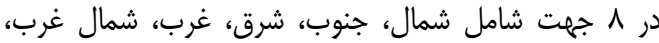

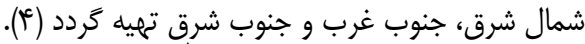
شاخص رطوبت تويوكرافى (TWI)': اين شاخص، شئرئ شاخص

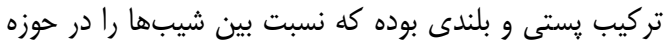

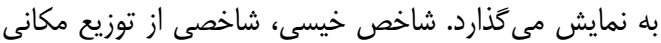

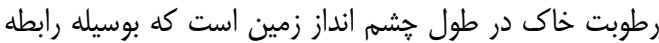

$T W I=\operatorname{In}\left(A_{S} / \tan \beta\right)$ ( اقابل محاسبه مىباشد (ج) (r).

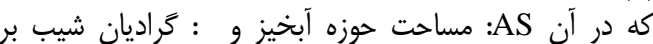

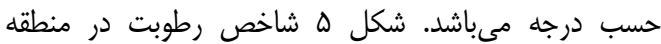
مطالعاتى را نشان مىدهد.

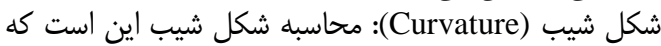

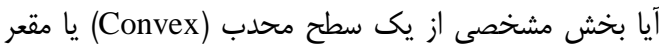

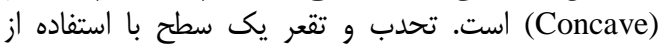

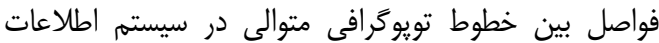

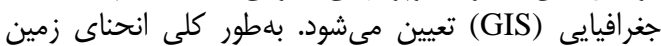
بين مقادير مثبت (محدب) و منفى (مقعر) در نوسان است أنساي 

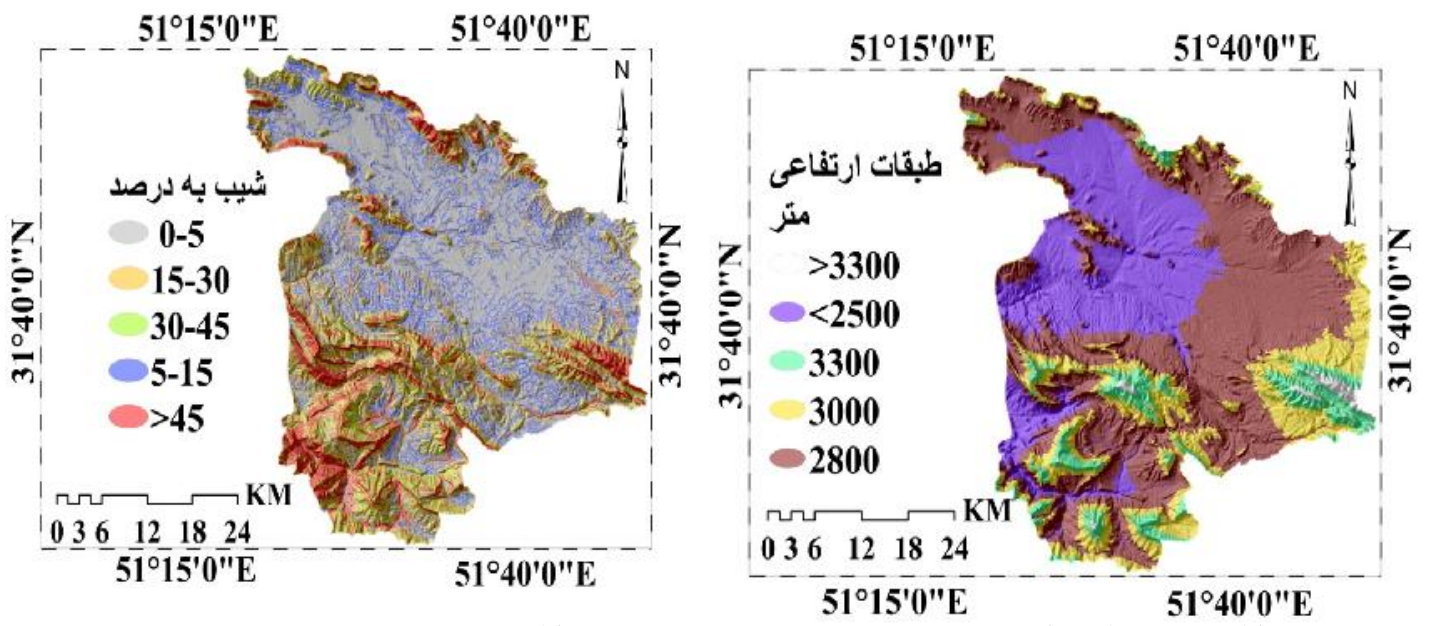

Figure3. Slope map

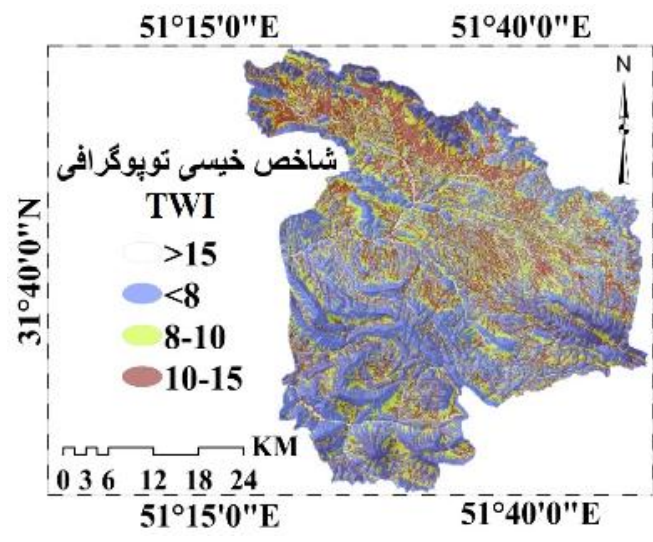

شكل ه- نقشه شاخص رطوبت

Figure 5. Topography wetness

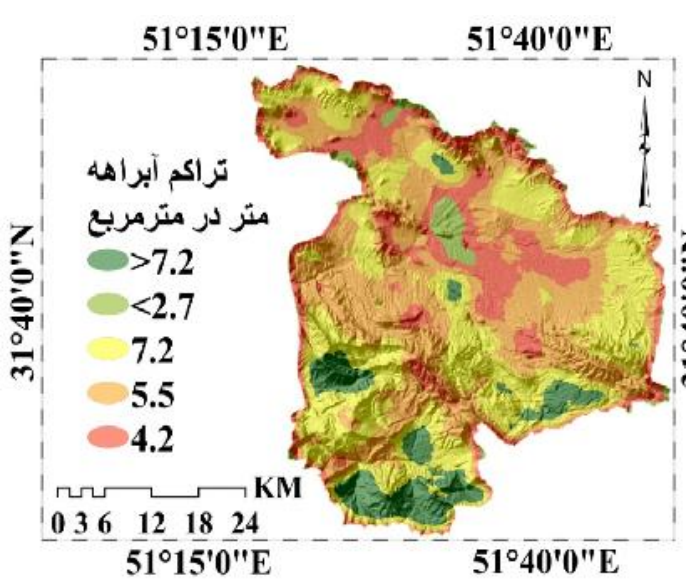

شكل V- نقشه تراكم آبراهـ

Figure 7. stream density map

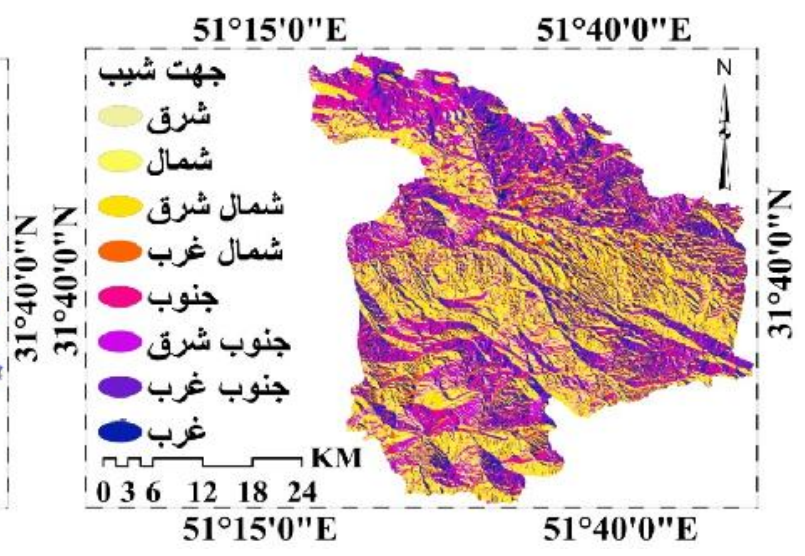

شكل عأ- نقشه جهت شيب

Figure 4. aspect map

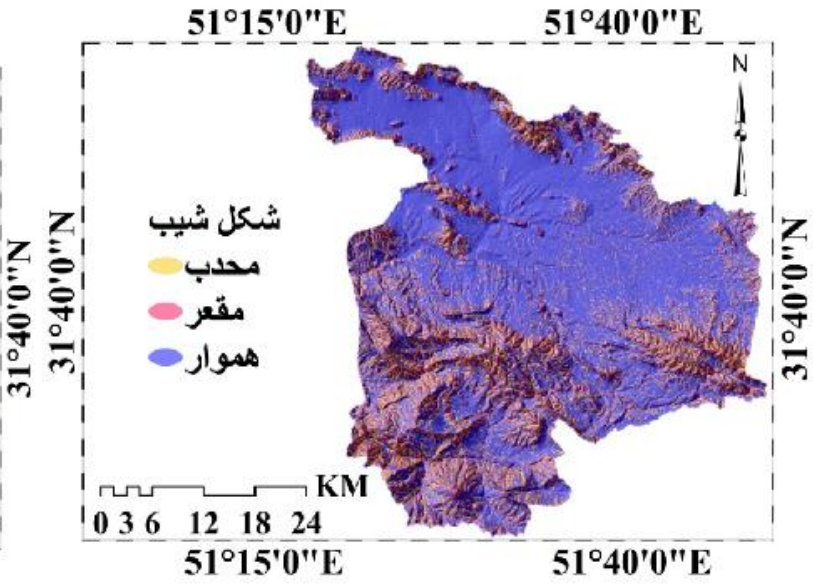

شكل و- نقشه شكل شيب

Figure 6. Plan curvature map index 


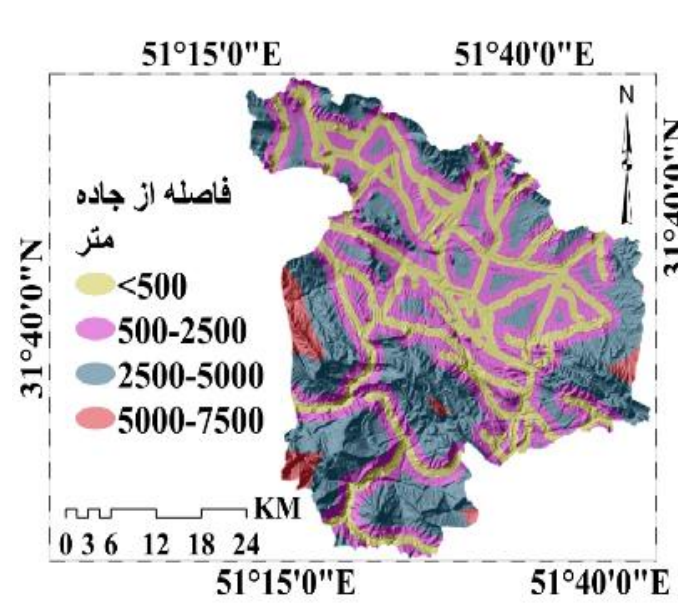

شكل q- نقشه فاصله از جاده

Figure 9. Distance from road

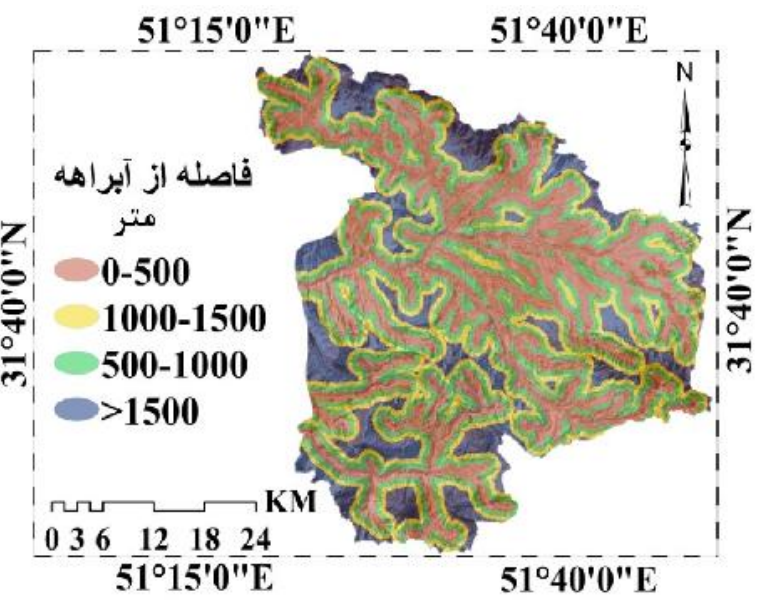

شكل ^- نقشه فاصله از آبراهـ

Figure 8. Distance from river

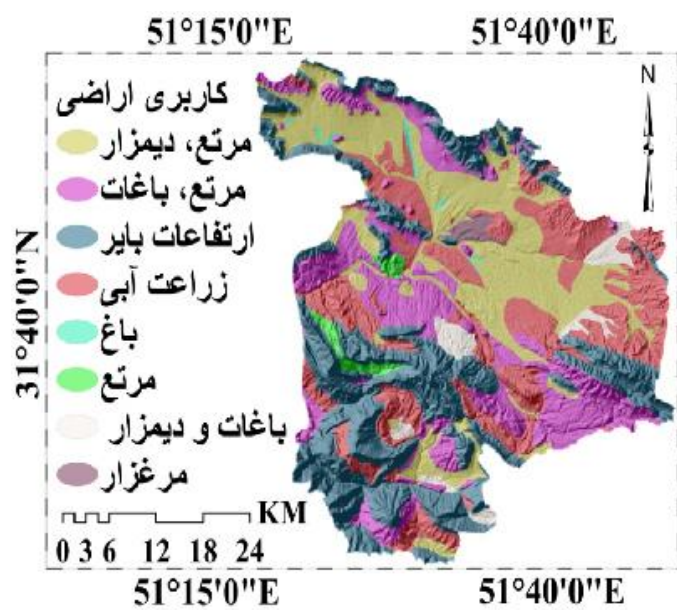

شكل II- نقشه كاربرى اراضى

Figure 11. Land use map

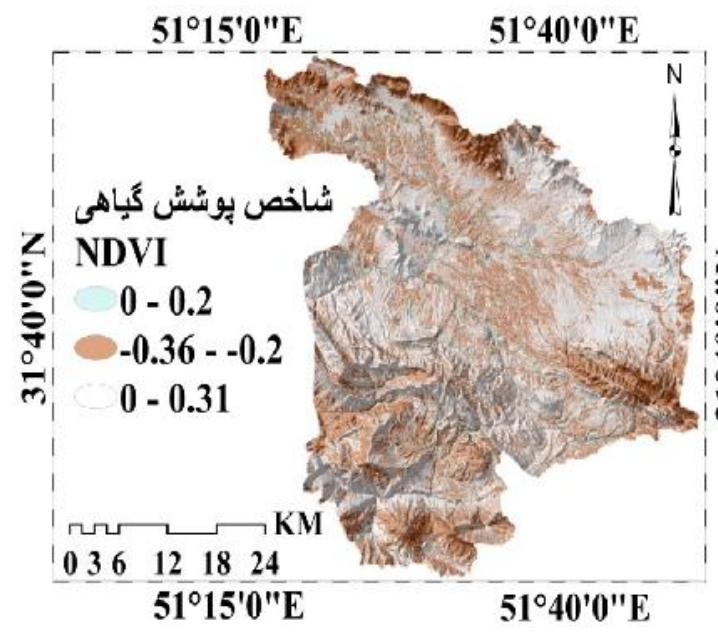

شكل سו- نقشه شاخص يوشش كَاهى

Figure 13. NDVI index

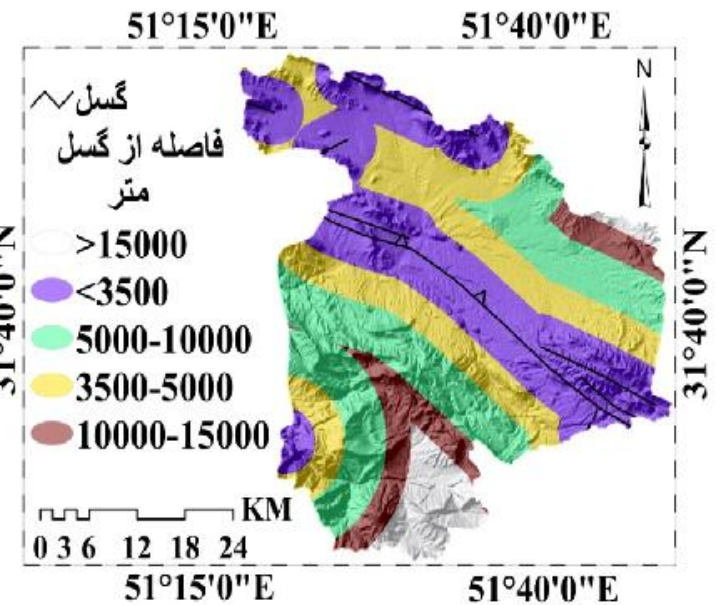

شكل • • - فاصله از كسل

Figure 10. Distance from fault

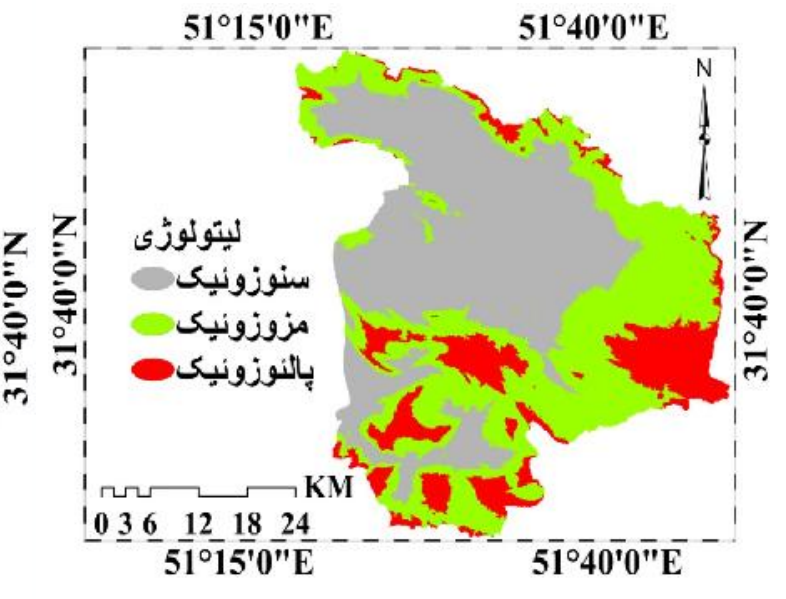

شكل r|- نقشه ليتولوزى

Figure 12. Lithology map 
دادههاى ماهوارهاى لندست هفت با استفاده از نرمافزار ENVI

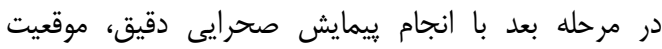

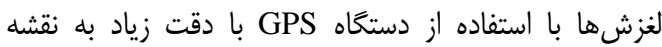

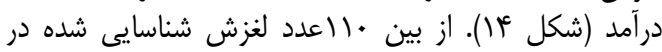

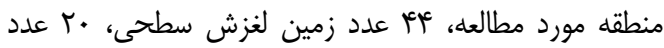

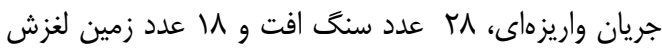

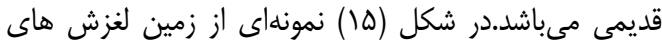
رخ داده در غرب ونك نمايش داده شده است.

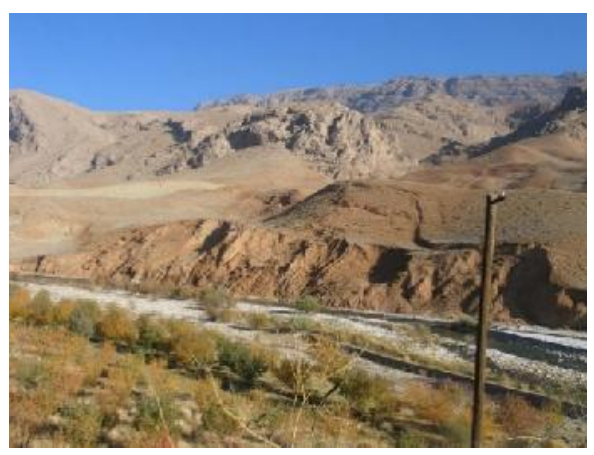

شكل Dا - زمين لغزش انتقالى در غرب ونك

Figure 15. transition Landslide in west of vanak
نقشه يراكنش زمين لغزشها

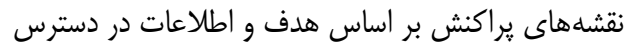

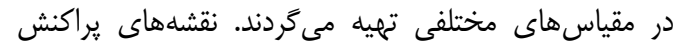

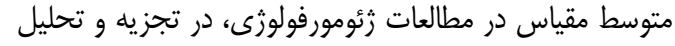

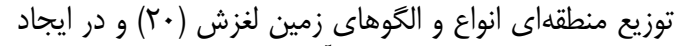

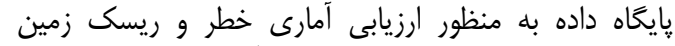

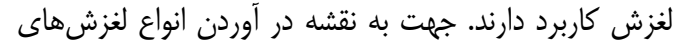

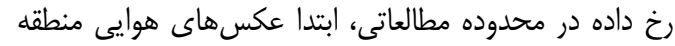

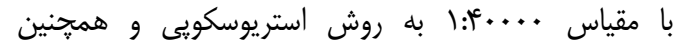

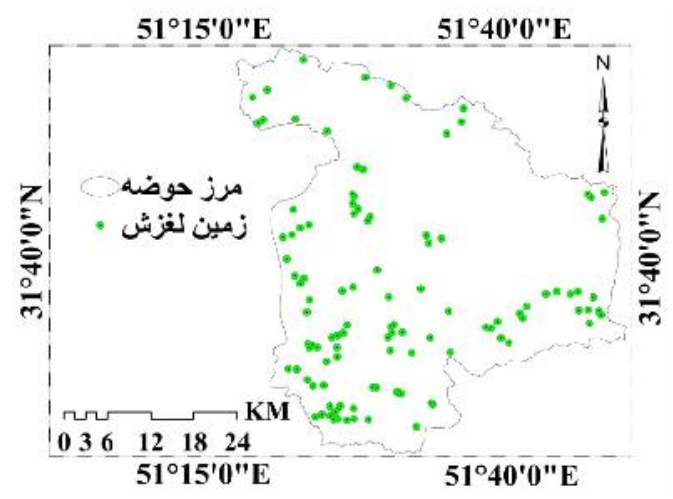

شكل عأ- نقشه يراكنش زمين لغزشها

Figure 14. Inventory map

\section{روش احتمالاتى وزن واقعه}

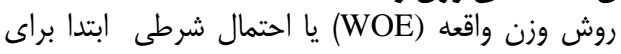

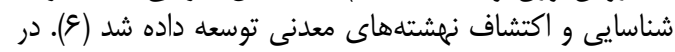

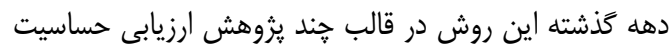

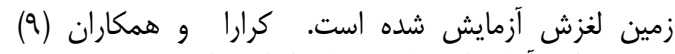
معتقدند كه آناليز احتمال شرطى آزايش شاره است ارزشمند در تعيين

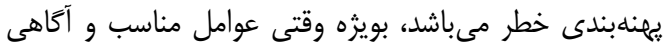

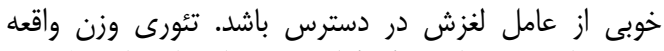

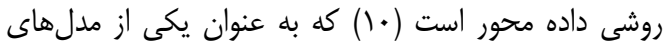

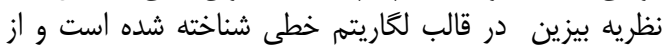

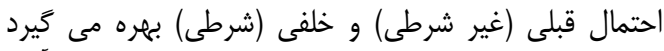

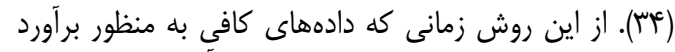

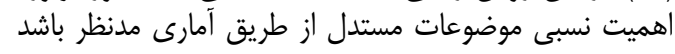

Table1. Four possible combinations of a potential landslide conditioning factor

جدول ا- جِهار حالت يتانسيل ايجاد لغزش (q")

\begin{tabular}{|c|c|c|}
\hline زمين لغزش & حضور & عدم حضور \\
\hline حضور & $N_{\text {pix } 1}$ & $N_{p i x 2}$ \\
\hline عدم حضور & $N_{p i x 3}$ & $N_{p i x 4}$ \\
\hline
\end{tabular}

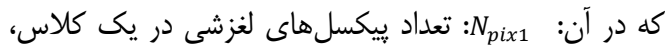
Noix2

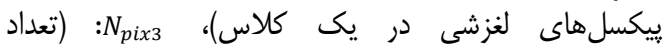

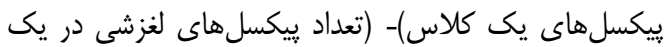
كلاس)،

$$
\begin{aligned}
& W_{i}^{+}=\log _{e} \frac{\left(N_{p i x 1} /\left(N_{p i x}+N_{p i x 2}\right)\right)}{\left(N_{p i x} /\left(N_{p i x}+N_{p i x 4}\right)\right)} \\
& W_{i}^{-}=\log _{e} \frac{\left(N_{p i x 2} /\left(N_{p i x 1}+N_{p i x 2}\right)\right)}{\left(N_{p i x} /\left(N_{p i x 3}+N_{p i x}\right)\right)}
\end{aligned}
$$


دارند، براى ارزيابى دقت تفكيك طبقات مدل استفاده

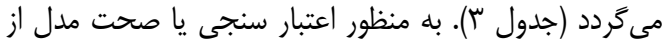

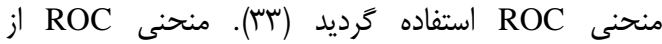

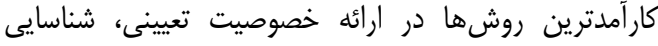

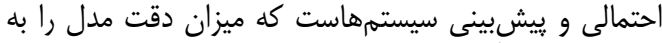

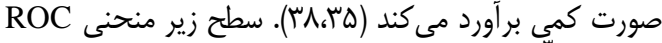

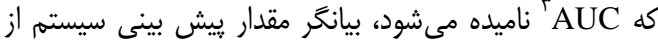

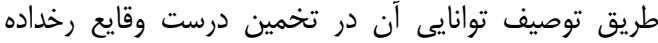

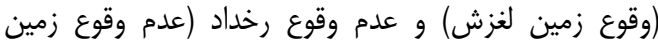

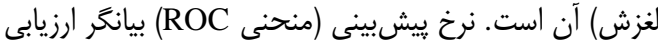

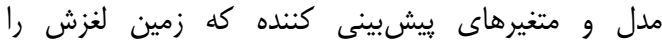
بيشيينى مى كند، مى مباشد (^).

\section{نتايج و بحث}

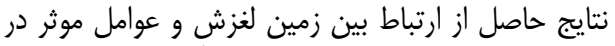

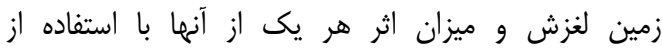

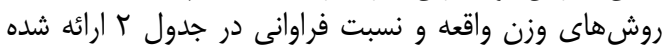

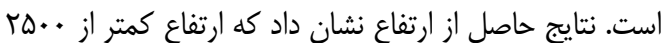

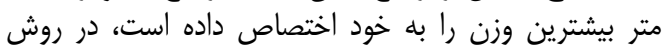

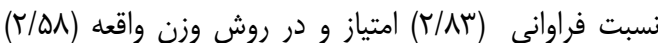

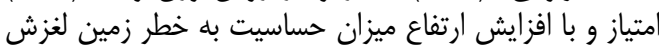

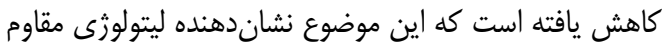

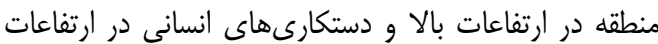

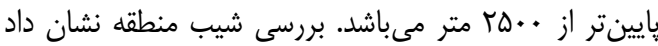

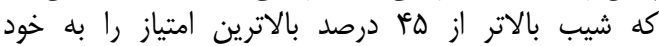

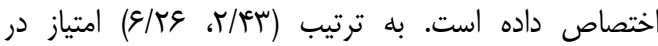

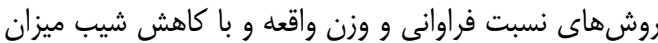

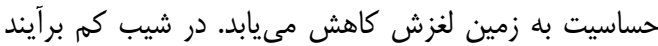

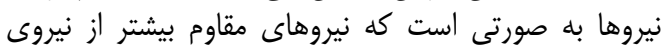

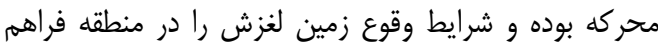

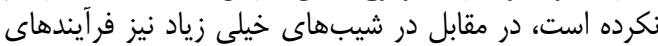

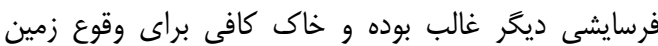

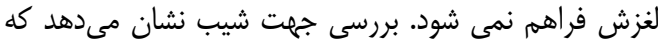

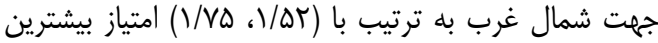

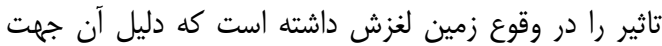

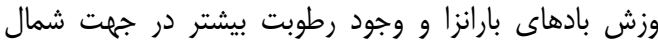

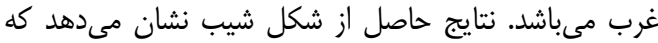

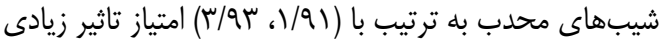

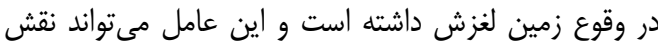

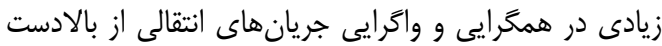

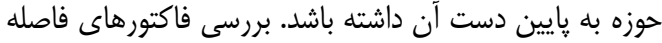

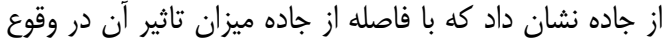

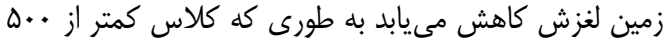

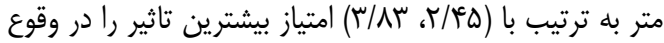

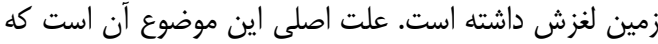

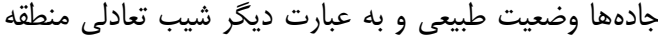

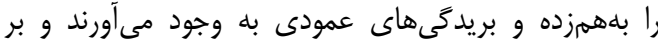

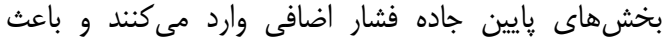

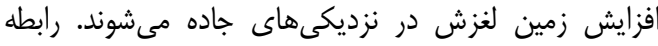

$$
\begin{aligned}
& \text { كل يبكسلهاى لغزشى يك نقشه)- (تعداد بيكسل هاى يك } \\
& C=\left[\left(W^{+}\right)-\left(W^{-}\right)\right] \\
& W_{\text {final }}=C / S_{C}
\end{aligned}
$$

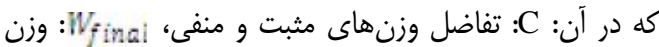

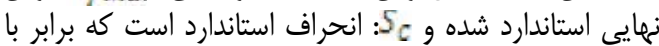

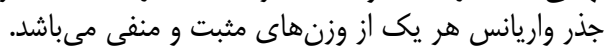

روش نسبت فراوانى

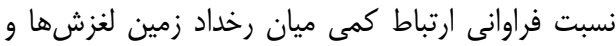

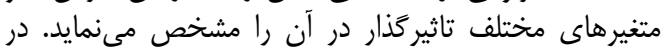

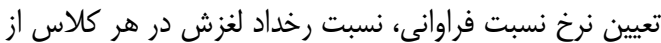

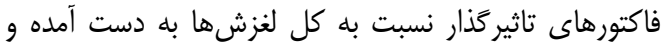

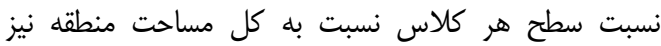

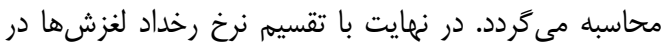

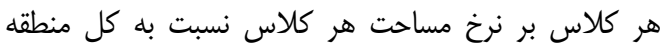

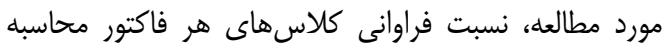

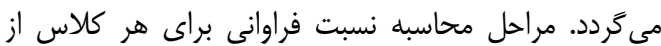
فاكتورهاى موثر در لغزش در رابطه ^ بيان شده است.

$F R=\frac{(A / B)}{(C / D)}=\frac{E}{F}$

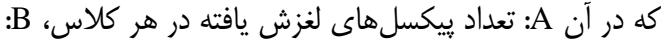

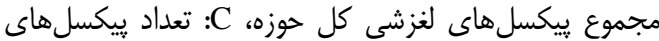

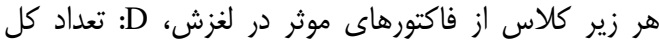

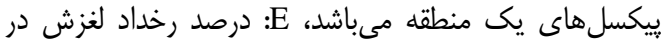

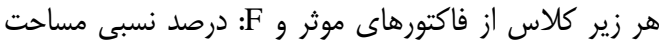

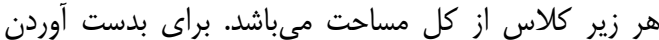

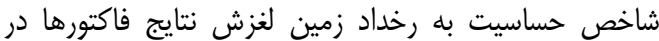

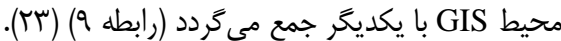

$L S I=\sum(F R)_{i} \quad(i=1,2, \ldots, n)$

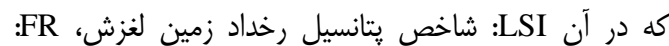

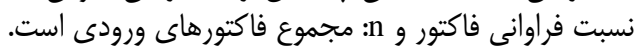

ارزيابى مدلها

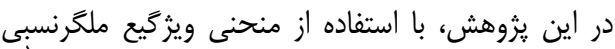

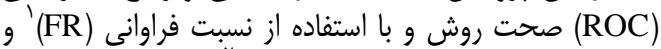

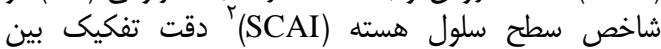

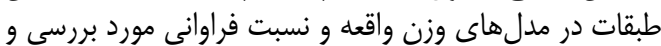

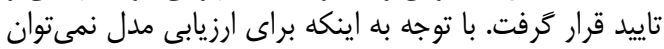

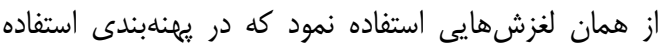

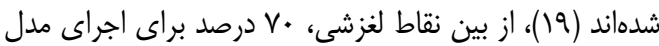

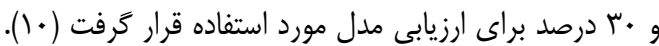

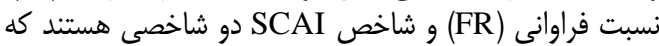

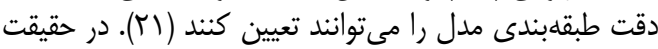

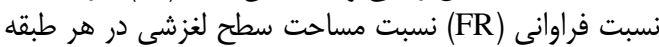

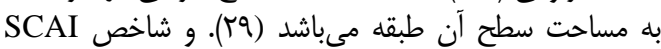

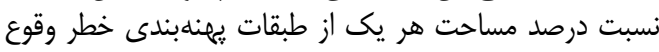

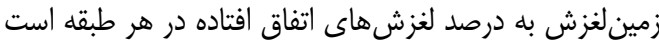

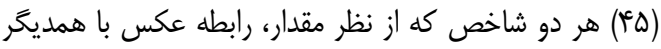




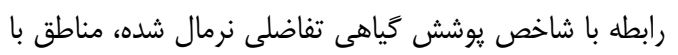

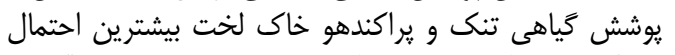

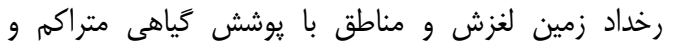

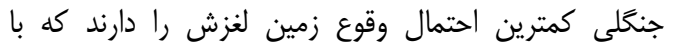

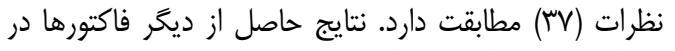
جدول r نشان داده شده است.

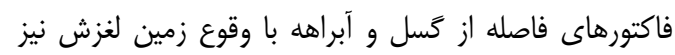

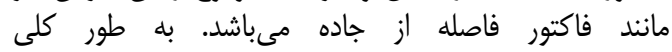

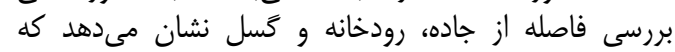

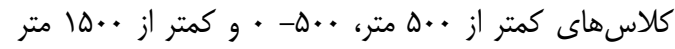

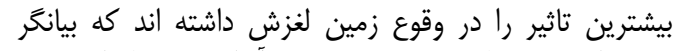

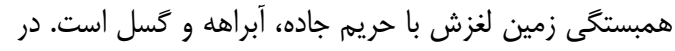

جدول ז- ارتباط بين هر يك از عوامل موثر و نقاط لغزشى با استفاده از مدل احتمالاتى وزن واقعه و نسبت فراوانى Table 2. Spatial relationship between each landslide conditioning factor and landslide by WOE and FR models

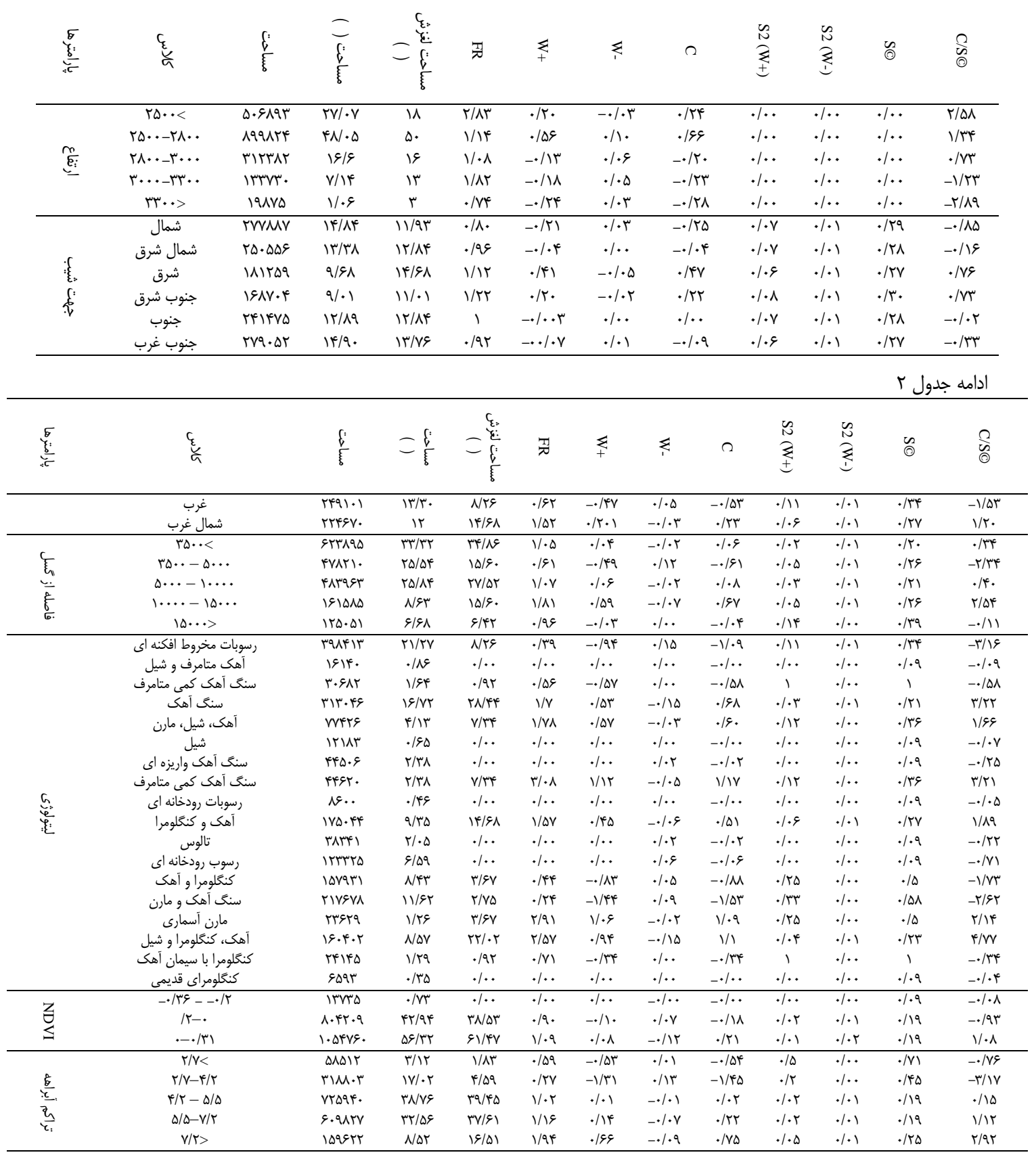




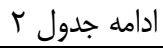

\begin{tabular}{|c|c|c|c|c|c|c|c|c|c|c|c|c|}
\hline$\frac{\xi}{\xi}$ & $\begin{array}{l}\xi_{\xi} \\
\text { L }\end{array}$ & $\begin{array}{l}\vdots \\
\xi \\
\xi\end{array}$ & $=\xi_{\xi}^{g}$ & $=\begin{aligned} & \frac{\varepsilon}{\xi} \\
&= \frac{\xi}{\xi} \\
& \xi\end{aligned}$ & 금 & $\sum$ & $\sum_{1}$ & $\Omega$ & $\begin{array}{l}\stackrel{N}{N} \\
\sum \\
\pm\end{array}$ & $\sum_{i}^{N}$ & $\widetilde{0}$ & $\frac{2}{\hat{n}}$ \\
\hline \multirow{8}{*}{ 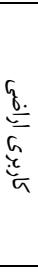 } & زراعت أبى، باغات، ديم & FTTDMA & $T r / \Delta V$ & N/TG & $\cdot / T V$ & $-1 / \cdot$ &.$/ 19$ & $-1 / I V$ &.$/ 11$ & $.1 \cdot 1$ & $\cdot / \pi F$ & $-r / \mu \Lambda$ \\
\hline & مرتع و ديمزار & TESAVT & N/QT & $\mid \omega / 9$ & $\cdot / \lambda F$ &.$- / 1 V$ & 少 & $-* / T$. &.$/ \cdot 1$ & $.1 \cdot 1$ & ع & $-\cdot / v 9$ \\
\hline & ارتفاعات باير، مرتع & $\Delta Q F I Q T$ & $r r / \Delta q$ & $\Delta V / \Lambda$. & $r / 91$ & .199 &.$-|0|$ & $1 / 11$ & .1 .4 & .1 .4 &.$/ 19$ & 91.9 \\
\hline & ديمزار، ارتفاعات باير & FEVADT & $r r / q)$ & $1 r / v q$ & $\cdot / \Delta \Lambda$ & $-\cdot / \Delta \Delta$ &.$/ 1 r$ & $-.19 \mathrm{~V}$ &.$/ \cdot 1$ & $.1 \cdot 1$ & $\cdot / T V$ & $-t / A^{2}$ \\
\hline & مرغزار، مرتع & $\Lambda \Delta M$ &.$/ 48$ & $\%$ & $\cdot / \cdot$ &.$/$ &.$/$. &.$- /$. &.$/$. &. & .1 .9 & -.1 .0 \\
\hline & مرتع، ديمزار & D D. D & $1 / T \&$ & $r / 9 V$ & $1 / 11$ & .1. &.$- / .4$ &.$/ .4$ &.$/ \cdot$ &. & .1 .9 & $\cdot / T \Delta$ \\
\hline & مرتع، باغات & $\Delta F \wedge .$. & r/q & . /9r &.$/ 41$ & $-1 / 10$ & .1 .4 & $-1 / 11$ & 1 &. & $1 / \cdot$ & $-1 / 1 V$ \\
\hline & زراعت آبى،باغات،مرتع & IfTES &.$/ V G$ & .1. &.$/$. & $\cdot / \cdot$ &.$/$. & $-* /$. & $\cdot / \cdot$ &. & .1 .9 & $-\cdot 1 \cdot 1$ \\
\hline \multirow{4}{*}{ 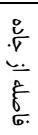 } & $\Delta \cdot \cdot<$ & 490949 & $r g / F \Lambda$ & $\Delta \& / \Lambda$ & $T / F \Delta$ & $-1 / \cdot 9$ & $\cdot|r|$ & $-1 / T V$ & $\cdot / 1$ &.$/ \cdot 1$ & אז/. & $r / \wedge r$ \\
\hline & $\Delta \cdots-r \Delta \cdots$ & SQIATF & $M F / \Lambda 1$ & $r \Delta / \bar{q}$ & $1 / 91$ &.$- / \mu \cdot$ & 每 & س & r & $\cdot|r|$ & $\cdot / r \mid$ & $-1 / 11$ \\
\hline & $r \Delta \cdots-\Delta \cdots$ & GQINET & $r \Delta / r f$ & 1 & $\cdot / V^{e}$ & $\cdot / \mathbb{E r}$ &.$- / f$ & $\cdot / M$ &.$/ \cdot 1$ & .119 &.$/ 19$ & $-1 / \Delta 9$ \\
\hline & $\Delta \cdots-v \Delta \cdots$ & 94.91 & $r / T r$ & N/TE & . &.$/ 19$ & $-.1 \cdot 0$ &.$/ 95$ &.$/ 11$ & F & . W & $-T / N T$ \\
\hline \multirow{5}{*}{$\xi$} & $\cdot-\phi$ & $\lceil\wedge \Delta \mid .$. & ro/q. & N/T\& & $\cdot / K r$ & $-1 / / f$ & $\cdot|r|$ & $-1 / \Gamma \Delta$ &.$/ 11$ & $.1 \cdot 1$ &.$/ \mu F$ & $-r / q$. \\
\hline & $\Delta-10$ & $r \Delta \cdot r \cdot r$ & $r F / \cdot \Delta$ & $I T / A F$ & / &.$- / 94$ & r &.$- / V G$ & $.1 . \mathrm{V}$ & .1 .1 &.$/ T \Lambda$ & $-\Gamma / Q V$ \\
\hline & $10-r$. & TVQIAD & $r \cdot / T \Delta$ & $\mid Q / 9$. & $\cdot / V V$ &.$- / T \&$ & $.1 \cdot 0$ & | & .1 .0 & .1 .1 & . & $-1 / r$. \\
\hline & $r \cdot-r_{\Delta}$ & 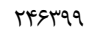 & $\mid r / 19$ & $1 r / 9 F$ & $1 / V^{e}$ & $\cdot / \Delta \Delta$ &.$- / 11$ &.$/ 9 \mathrm{~V}$ & .1 .4 & .1 .1 & T & r/q६ \\
\hline & $\langle\Delta\rangle$ & mIleiv & $\mid g / 9 T^{\circ}$ & $r \cdot / r v$ & T/F & $\cdot / \mathrm{M}$ & سז/.- & I/Tr &.$/ .4$ & $.1 \cdot 1$ & .119 & g/re \\
\hline \multirow{4}{*}{$\xi$} & $<\Lambda$ & $99991 \%$ & Q1/GT & $98 / 14$ & $1 / R q$ & ( & 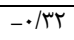 & $\cdot / \Delta \Delta$ &.$/ \cdot 1$ &.$/ . r$ & $\cdot / r$. & $r / v q$ \\
\hline & $\Lambda-1$. & EAVGVT & $r F / q r$ & $T r / \cdot r$ & $\cdot / M$ &.$- / 1 T$ & . &.$- / 19$ & $.1 \cdot 4$ & $.1 \cdot 1$ & سז/. & $-\cdot / V 1$ \\
\hline & 1. -10 & 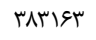 & $r . / 49$ & $9 / 1 V$ & $\cdot / 4 \Delta$ & $-\cdot / 1$ & r & r/9 &.$/ 1$ & $.1 \cdot 1$ & אץ/. & $-r / A r$ \\
\hline & $|Q\rangle$ & $\Delta F q \Delta F$ & $r / Q Y Y F$ & $r / 999$ & $1 / T \Delta$ & 政 &.$- / . \cdot$ & Tr/ &.$/ T \Delta$ & .1. & $\cdot / 0$. & سזא/. \\
\hline \multirow{3}{*}{$\begin{array}{l}\xi \\
\bar{\xi} \\
\underline{\xi}\end{array}$} & مقعر & TAVDIA & $\mid \Delta / r \Delta$ & rq/rq & $1 / T \Delta$ & - /at & $-\cdot / 11$ & ./AT & $\cdot / \cdot r$ &.$/ .1$ & $\cdot|r|$ & $r / q \varphi$ \\
\hline & هموار & ITQDT.D & sV/.r & FN/GT & $\cdot / N$ & $-. \cdot / r T$ & $\cdot / 4 F$ & $-\cdot / v 9$ &.$/ \cdot 1$ & $.1 \cdot 1$ & $.1 / 9$ & $-r / 99$ \\
\hline & محدب & тт9911 & IV/GT & $T r / .4$ & $1 / 91$ &.$/ 4 \pi$ &.- .1 .0 & $\cdot / r V$ & .1 .4 & .1 .1 & سr/. & r/q \\
\hline \multirow{4}{*}{ 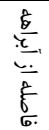 } & $\cdot-\Delta \cdots$ & $V \cdot 1 . . r$ & $T V / F T$ & $r \Delta / v \wedge$ &.$/ 98$ & $-\cdot / .4$ &.$/ \cdot r$ & $-* / \cdot V$ &.$/ .4$ & $.1 \cdot 1$ &.$/ 19$ & G \\
\hline & $\Delta \cdots-1 \cdots$ & FА१人. & re/IS & $19 / T r$ & $\cdot / V F$ &.$- / \Gamma$ & $\cdot 1 \cdot 1$ &.$- / 49$ & .1 .4 & $.1 \cdot 1$ & $\cdot / M F$ & س \\
\hline & $1 \cdots-10 \cdots$ & MTFEgq & IV/r Tr & $10 / 9$ &.$/ 9$. & $-0 / 1$ & .1 .4 &.$- / T$ & .1 .0 & $.1 \cdot 1$ & G &.$- / 4 \lambda$ \\
\hline & $>10 .$. & roVrqT & $19 / \cdot 1$ & rq/זq & $1 / \Delta F$ & Tא/. & سו/.-. & $\cdot / \Delta S$ & r & $.1 \cdot 1$ & $\cdot|r|$ & $r / q q$ \\
\hline
\end{tabular}

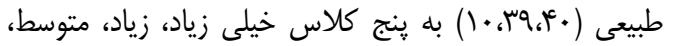

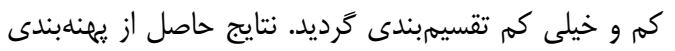
خطر زمين لغزش با استفاده از روش نسبت فرديت فراوانى در شكل (19) و با روش وزن واقعه در شكل (IV) نشان داده شده ندان

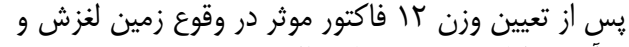

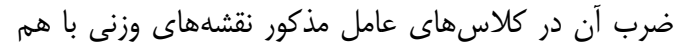

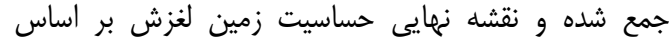

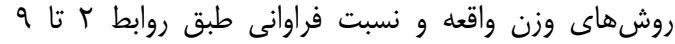
حاصل گرديل، سبس نقشه مذكور بر اساس شكستهاى روائ

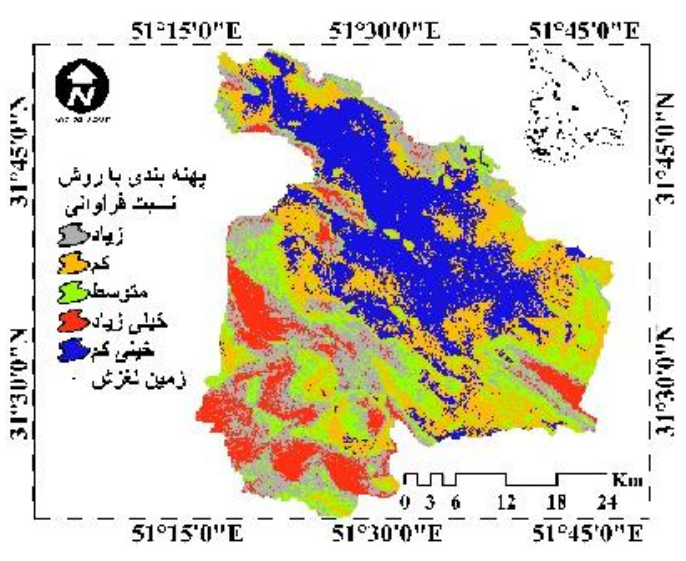

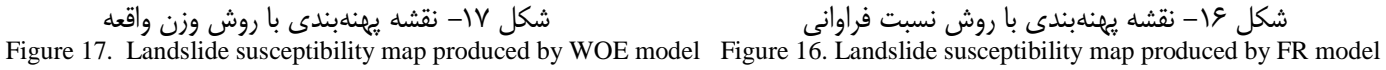

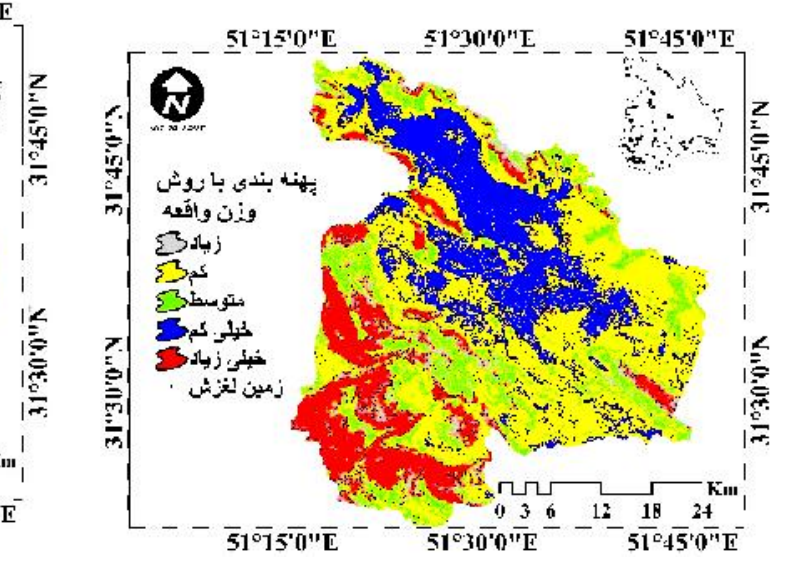


تفكيكيذيرى بين طبقات، مناسب ارزيابى گرديد. اين

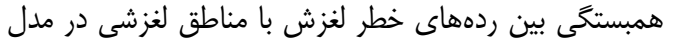

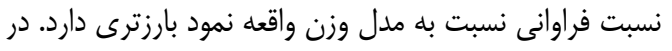

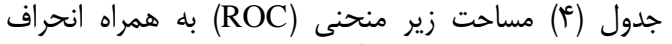

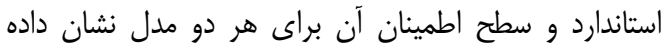

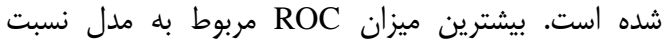

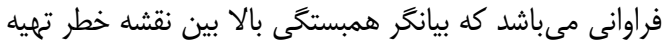

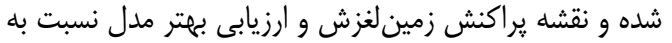

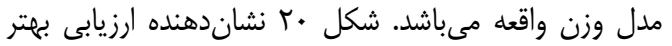

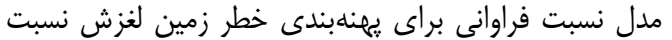
به مدل وزن واقعه مىباشد.

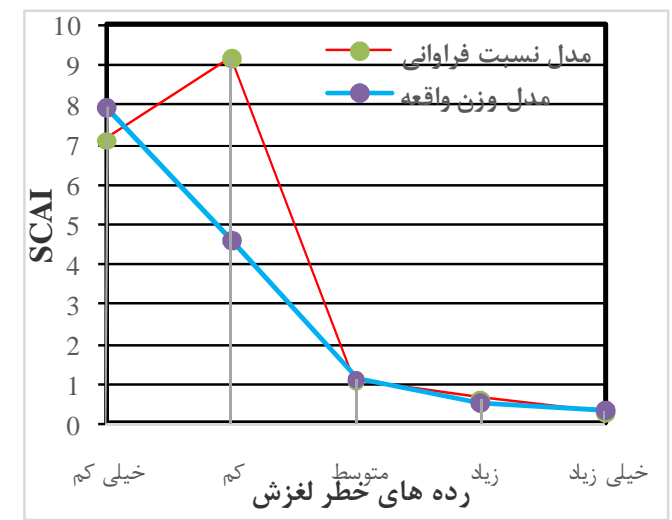

شكل 19- روند شاخص FR در مدلها

Figure 19. Trends of FR Index in models
يس از تهيه نقشههاى يهنهبندى خطر زمين لغزش اقدام به

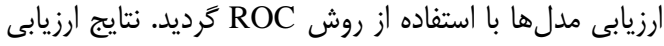

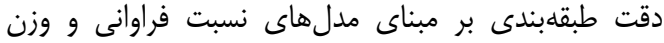

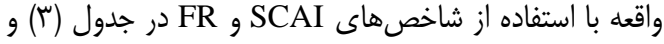

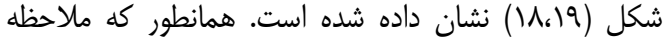

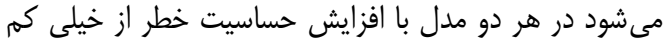

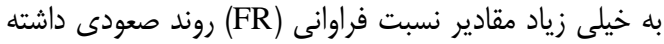

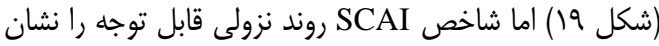

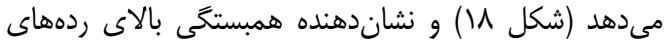
خطر لغزش با مناطق لغزشى موجود و و بازديدهاى مناى ميدانى

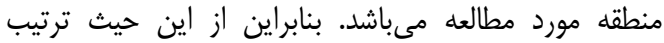

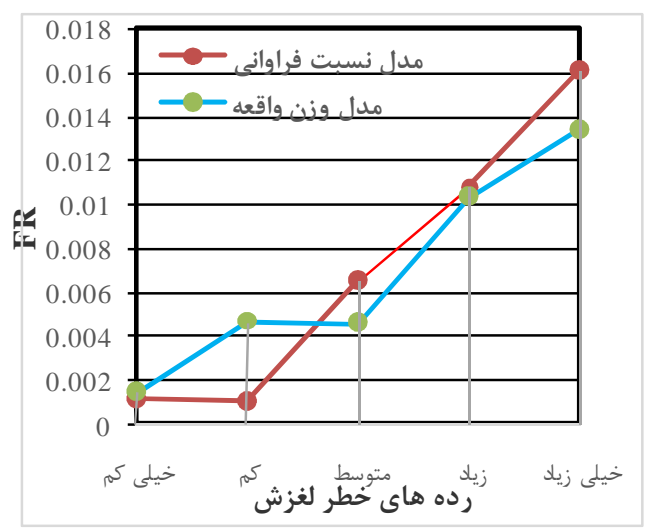

شكل 11- روند شاخص SCAI در مدلها

Figure 18. Trends of SCAI Index in models

Table 3. FR and SCAI for the landslide susceptibility map

جدول س- نسبت فراوانى (FR) و شاخص SCAI يهنهبندى خطر وقوع زمين لغزش

\begin{tabular}{|c|c|c|c|c|c|c|c|c|}
\hline$\tilde{\Sigma}^{n}$ & 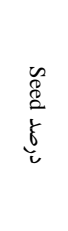 & 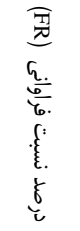 & $\begin{array}{l}\stackrel{8}{0} \\
\delta \\
\delta \\
\delta \\
\delta \\
\zeta\end{array}$ & 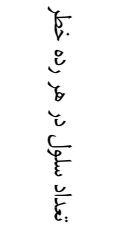 & $\begin{array}{l}\varepsilon_{:} \\
\dot{\varepsilon} \\
\check{\check{\varepsilon}} \\
\zeta \\
\zeta\end{array}$ & 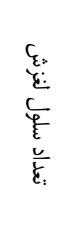 & 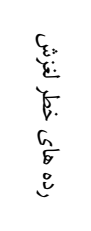 & $\bar{\xi}$ \\
\hline $\mathrm{V} / \mathrm{II}$ & $r / T \Delta$ &.$/ . \cdot 1 r$ & $r \mu / .9$ & 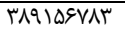 & $F / \Delta q$ & $r \Delta \cdot$. & خيلى كم & \\
\hline $9 / 1 \wedge$ & $r / \Lambda \varepsilon$ &.$/ .1$ & TE/TE & FEYMA-DQF & $5 / 19$ & $r \Delta .$. & كم & \\
\hline $1 / \cdot v$ & W/Ta &.. .90 & 19/8 & & $T r / \cdot T$ & MIs.. & متوسط & نسبت فراوانى \\
\hline . & $r \cdot / \mu f$ & $\cdot 1 \cdot 1 \cdot 1$ & IN/VA & MISQVATAF & $\mu \varphi / N E$ & MFY.. & زياد & \\
\hline$\cdot / T V$ & $F \Delta / r$. & .1 .191 & $I T / T A$ & $r \cdot 99 \cdot M \mu \wedge$ & rr/qY & . . ז"זץ & خيلى زياد & \\
\hline$s / V$. & $F / 11$ &.$/ . .1 f$ & TV/OT & $F \& \Delta \wedge r F+q$. & $\varepsilon / \& V$ & sQTq & خيلى كم & \\
\hline $1 / A \varepsilon$ & $\mid r / F \lambda$ & $.1 .+48$ & $r \Delta / 1 Q$ & 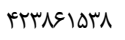 & $r$. & 1995. & كم & \\
\hline $1 / T V$ & $\mid r / r v$ & $.1 .+49$ & $18 / 91$ & TAD. $90 \wedge 99$ & אس/או & $1 \pi \cdot \Lambda \cdot$ & متوسط & وزن واقعه \\
\hline س T/. & $r .1 .1$ & $.1 \cdot 1 \cdot 4$ & W/AT & TIVETMIFI & איז/ & rrsq9 & زياد & \\
\hline$\cdot / \mu$. & rq/.r & $.1 .1 \% f$ & $11 / \Delta A$ & 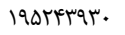 & $r q / 9 V$ & r9ו9. & خيلى زياد & \\
\hline
\end{tabular}

Table 4. Area under the curve (AUC) with Standard deviation

جدول عا- مساحت زير منحنى (ROC) به همراه انحراف استاندارد

\begin{tabular}{|c|c|c|c|c|c|}
\hline \multirow{2}{*}{ TestResult Variable(s) } & \multirow{2}{*}{ Area } & \multirow{2}{*}{ Std. Error ${ }^{a}$} & \multirow{2}{*}{$\begin{array}{c}\text { Asymptotic } \\
\text { Sig. }\end{array}$} & \multicolumn{2}{|c|}{ Asymptotic $95 \%$ Confidence Interval } \\
\hline & & & & Lower Bound & Upper Bound \\
\hline مدل نسبت فراوانى & $\cdot / V^{\mu} F$ & $\cdot . \cdot \omega$ & $\cdot / \cdot \cdot r$ & .1990 & $\cdot / \Lambda \cdot r$ \\
\hline مدل وزن واقعه & . & . $1 . \% 9$ &.$\%$ & . DAF & $\cdot / \mathrm{V} \cdot$ \\
\hline
\end{tabular}




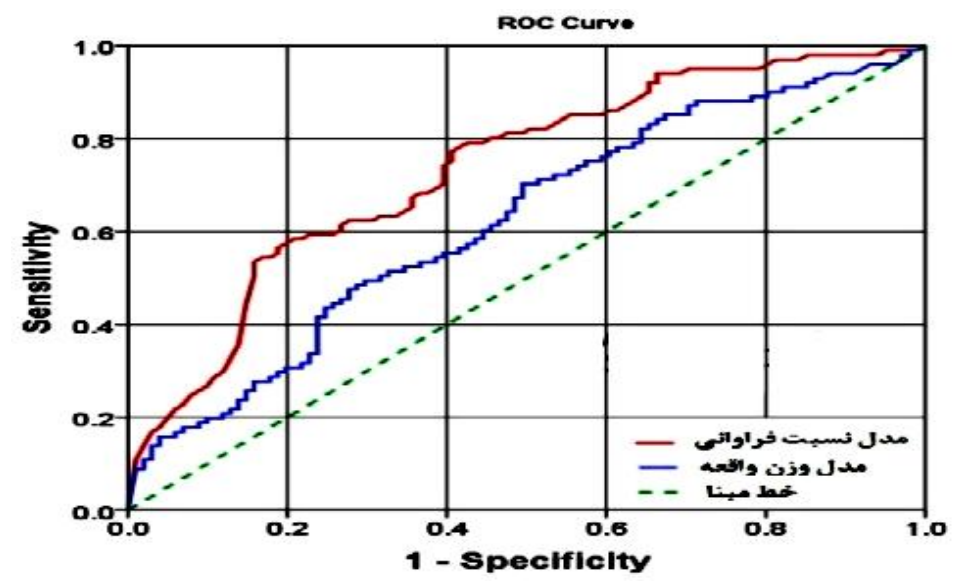

(ROC) شكل • (r- منحنى نرخ يِيشينى

Figure 20. Receiver Operating Characteristics

(qس•/•) و امتياز (سبع/•) جهت ارزيابى زمين لغزشها در

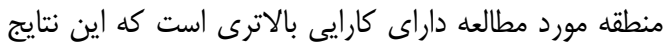

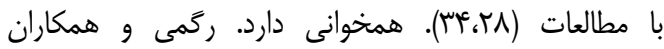

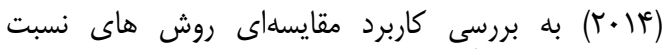

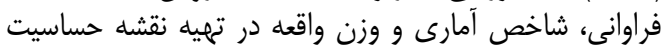

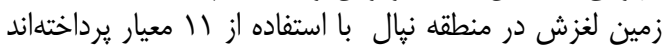

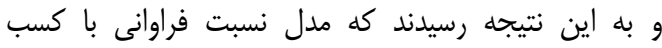

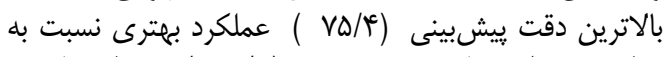

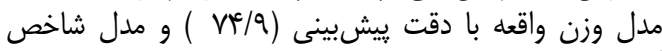

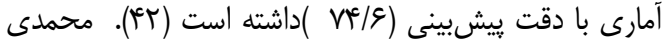

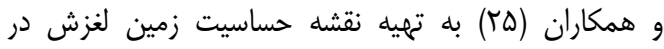

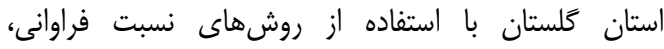

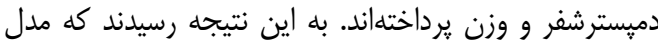

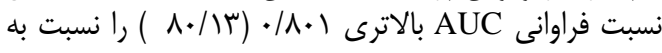

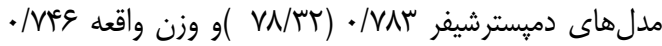

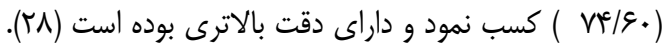

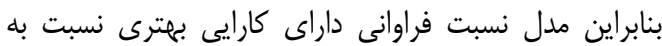

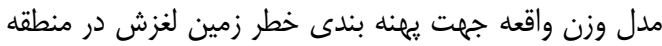

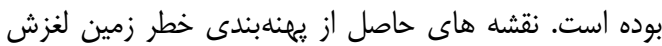

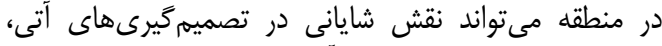

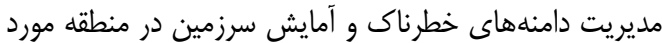

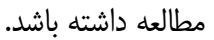

هدف از تحقيق حاضر اولويتبندى عوامل موثر در وقوع

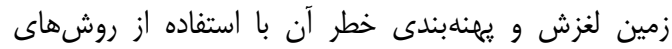

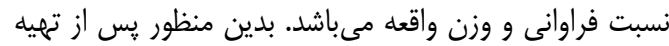

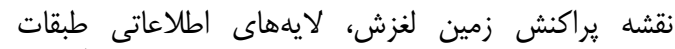

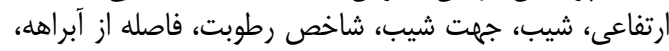

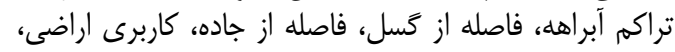

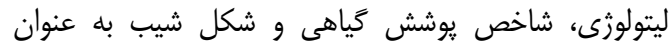

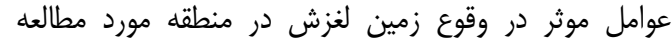

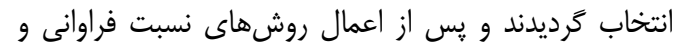

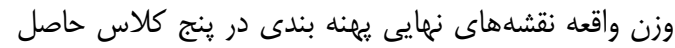

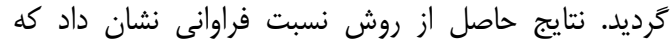

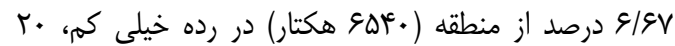

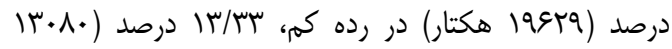

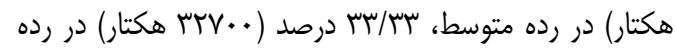

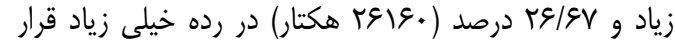

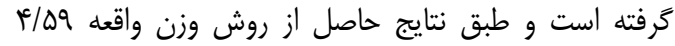

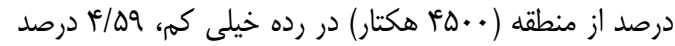

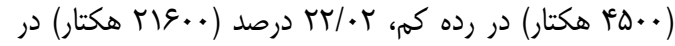

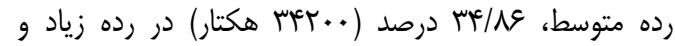

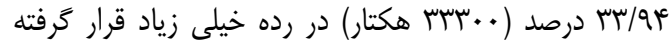

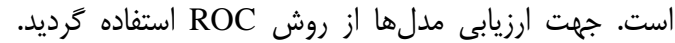

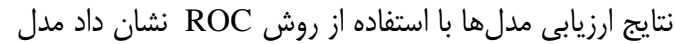

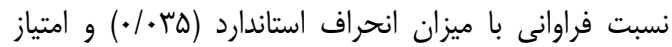

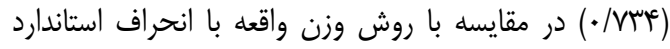


1. Arabameri, A.R A. Klorajan, J. Karami, M. Alimoradi and K Shirani. 2014 Zonation of Landslide Hazard Using Artificial Neural Network the Case Study: Marbor Basin. Geodynamics Research International Bulletin, 03: 44-59 (In Persian).

2. Arabameri, A.R. and K. Shirani. 2014. An Evaluation of Area Density and Certainty Factor methods in Landslide hazard analysis: A Case Study: Vanak Basin. Geodynamics Research International Bulletin, 04: 112-128 (In Persian).

3. Arabameri, A.R. and A.H. Halabian. 2016. Landslide Hazard Zonation Using Statistical Model of AHP (Case Study: Zarand Saveh Basin). Physical Geomorphology, 28: 65-86 (In Persian).

4. Ayalew, L. and H. Yamagishi. 2005. The application of GIS-based logistic regression for landslide susceptibility mapping in the kakuda-yahiko Moun-tains, central Japan. Geomorphology, 65: 15-31.

5. Atkinson, P.M. and R. Massari. 1998. Generalized Linear modelling of Landslide Susceptibility in the Central Apennines, Italy. Computer Geoscience, 24: 373-385.

6. Bonham-Carter, G.F. 1994. Geographic Information Systems for Geoscientists: Modeling with GIS. Pergamon Press, Canada, 398 pp.

7. Bonham-Carter, G.F., F.P. Agterberg and D.F Wright. 1989. Weights of evidence modeling: a new approach to mapping mineral potential. In: Agterberg, F.P. and Bonham-Carter, G.F. (eds.) Statistical applications in the Earth Science, Geological Survey of Canada Paper 89-9, Ottawa, Canada: 171-183.

8. Bui, H.B., Q. Nguyen and V.T. Nguyen. 2008. GIS-based weight of evidence modeling for landslide susceptibility mapping at Jaechon area, Korea. Journal of International Symposium on Geo informatics for Spatial Infrastructure Development in Earth and Allied Sciences, 4: 64-68.

9. Carrara, A., G.B. Crosta and P. Frattini. 2003. Geomorphologic and historical data in assessing andslide hazard. Earth Surface Processes and Landforms, 28: 1125-1142.

10. Constantin, M., M. Bednarik, M.C. Jurchescu and M. Vlaicu. 2011. Landslide susceptibility assessment using the bivariate statistical analysis and the index of entropy in the Sibiciu Basin (Romania). Environment. Earth Sci, 63: 397-406.

11. Constantin, M., M. Bednarik, M.C. Jurchescu and M. Vlaicu. 2010. Landslide susceptibility assessment using the bivariate statistical analysis and the index of entropy in the Sibiciu Basin (Romania). Environmental Earth Science, 2: 397-406.

12. Dai, F.C. and C.F. Lee. 2002. Landslide characteristics and slop instability modeling using GIS, Lantau Island, Hong Kong. Geomorphology, 31: 181-216.

13. Dai, F.C. and C.F. Lee. 2001. Terrain-based mapping of landslide susceptibility using a geographical information system: a case study. Canadian Geotechnical Journal, 38: 911-923.

14. Dahal, R.K., S. Hasegawa, A. Nonomura, M. Yamanaka, S. Dhakal and P. Paudyal. 2008. Predictive Modelling of Rainfall-induced Landslide Hazard in the Lesser Himalaya of Nepal Based on Weightsof-evidence. Geomorphology, 102: 496- 510.

15. Das, I., S. Sahoo, C. Van Westen, A. Stein and R. Hack. 2010. Landslide susceptibility assessment using logistic regression and its comparison with a rock mass classification system, along a road section in the northern Himalayas (India). Geomorphology, 114: 627-637.

16. Ebrahimi, E., K. Solaimani and H.R. Pourghasemi. 2016. Evaluating the Efficiency of Probabilistic Weight of Evidence Model for Landslide Susceptibility Mapping (Case Study: Siyahbisheh Watershed, Mazandaran). Journal of Watershed Management Research, 7: 69-77 (In Persian).

17. Garfi, G. and D.E. Bruno. 2007. Fan morph dynamics and slope instability in the Mucone River Basin (Sila Massif, Southern Italy): signification of weathering and role of land use changes. Catena, 50: 181-196.

18. Gorsevski, P.V., P. Jankowski and P.E. Gessler. 2006. Heuristic Approach for Mapping Landslide Hazard Integrating Fuzzy Logic with Analytic Hierarchy Process Control and Cybernetics, 35: 121146.

19. Gökceoglu, C. and H. Aksoy. 1996. Landslide Susceptibility Mapping of the Slopes in the Residual Soils of the Mengen Region (Turkey) by Deterministic Stability Analyses and Image Processing Techniques. Engineering Geology, 44: 147-161.

20. Guzzetti, F., A.C. Mondini, M. Cardinali, F. Fiorucci, M. Santangelo and K.T. Chang. 2012. Landslide inventory maps: New tools for an old problem. Earth-Science Reviews, 112: 42-66.

21. Kayastha, P., M.R. Dhital and F.D. Smedt. 2012. Landslide susceptibility mapping using the weight of evidence method in the Tinau watershed, Nepal. Natural Hazards, 63: 479-498.

22. Komac, M. 2006. A landslide susceptibility model using the analytical hierarchy process method and multivariate statistics in per alpine Slovenia. Geomorphology, 74: 17-28.

23. Lee, S. and B. Pradhan. 2007. Landslide hazard mapping at Selangor, Malaysia using frequency ratio and logistic regression models. Landslides, 4: 33-41.

24. Lee, S. and J. Choi. 2004. Landslide susceptibility mapping using GIS and the weight-of-evidence model. Intl. Journal of Geographical Information Science, 18: 789- 814.

25. Mohammady, M., H.R. Pourghasemi and B. Pradhan. 2012. Landslide susceptibility mapping at Golestan Province, Iran: A comparison between frequency ratio, Dempster-Shafer and weights-ofevidence models. Journal of Asian Earth Sciences, 61, 221-236.

26. Mohammady, M., H.R. Pourghasemi, B. Neuhauser and B. Terhorst. 2007. Landslide Susceptibility Assessment Using Weights-of-evidence, Applied to a Study Area at the Jurassic Escarpment (SWGermany). Geomorphology, 86: 12- 24. 
27. Mosavi, SM., M. Abedini, A. Esmali and F. Madani. 2016. Landslide hazard Zonation by using Fuzzy MCDM Models in the GIS (Case Study: Izeh Urban Watersheds of Khuzestan). Journal of Watershed Management Research, 7: 78-87 (In Persian).

28. Mathew, J., V.K. Jh and G.S. Rawat. 2007. Weights of evidence modeling for landslide hazard zonation mapping in part of Bhagirathi valley. Uttarakhand. Current Science, 92: 628-638.

29. Nefeslioglu, H.A., T.Y. Duman and S. Durmaz. 2008. Landslide susceptibility mapping for a part of tectonic Kelkit Valley (Easten Black Sea Region of Turkey), Geomorphology, 94 : 401-418.

30. Pradhan, B. 2012. Landslide susceptibility mapping at Golestan Province, Iran: A comparison between frequency ratio, Dempster-Shafer and weights-of-evidence models. Journal of Asian Earth Sciences, 61: 221-236.

31. Pradhan, B. 2010. Remote sensing and GIS-based landslide hazard analysis and cross-validation using multivariate logistic regression model on three test areas in Malaysia. Advances in Space Research, 45: 1244-1256.

32. Pourghasemi, H.R., B. Pradhan, H.R. Moradi, M. Mohammady and M. Bednarik. 2013. A Comparative Assessment Between Index of Entropy, Logistic Regression and Frequency Ratio Models for Landslide Susceptibility Mapping in Iran, Natural Disasters, 4: 1-30.

33. Regmi, N.R., J.R. Giardino and J.D. Vitek. 2010. Modeling susceptibility to landslides using the weight of evidence approach: Western Colorado, USA, Geomorphology, 115: 172-187.

34. Regmi, A.D., K.C. Devkota, K. Yoshida, B. Pradhan, H.R. Pourghasemi, T. Kumamoto and A Akgun. 2014. Application of frequency ratio, statistical index, and weights-of-evidence models and their comparison in landslide susceptibility mapping in Central Nepal Himalaya. Arabian Journal of Geosciences, 7: 725-742.

35. Roering, J.J., J.W. Kirchner and W.E. Dietrich. 2005. Characterizing Structural and Lithology Controls on Deep-seated Land sliding: Implications for Topographic Relief and Landscape Evolution in the Oregon Coast Range. Geological Society of America Bulletin, 117: 654-668.

36. Song, K.Y., J. Oh, J. Choi, I. Park, C. Lee and S. Lee. 2012. Prediction of landslides using ASTER imagery and data mining models. Advances in Space Research, 49: 978-993.

37. Shirani, K. and A.R. Arabameri. 2015. Landslide Hazard Zonation Using Logistic Regression Method (Case Study:Dez-e-Oulia Basin), Journal of Science \& Technology Agricultural \& Natural Resource., Water and Soil Science., Isfahan University Technology, Isfahan, Iran, 72: 321-334 (In Persian).

38. Swets, J.A. 1988. Measuring the accuracy of diagnostic systems. Science, 240: 1285-1293.

39. Van Western, C.J. 2002. Use of weights of evidence modeling for landslide susceptibility mapping, Natural Hazards, 25: 1-21.

40. Varnes, D.J. 1984. Landslide hazard zonation: A review of Principes and Practice, UNESCO, France, $63 \mathrm{pp}$.

41. Wu, W. and R.C. Sidle. 1995. A distributedslope stability model for steep forested basins. Water Research, 31: 2097-2110.

42. Wati, S., T. Hastuti, S. Wijojo and F. Pinem. 2010. Landslide Susceptibility Mapping with Heuristic Approach in Mountainous Area, A Case Study in Tawangmangu Sub District, Central Java, Indonesia, International Archives of the Photogrammetry, Remote Sensing and Spatial Information Science, 38: 248-253.

43. Yalcin, A. 2008. GIS-based landslide susceptibility mapping using analytical hierarchy rocess and bivariate statistics in Ardesen (Turkey): Comparisons of results and confirmations. Catena, 72: 1-12.

44. Yesilnacar, E.K. 2005. The Application of Computational Intelligence to Landslide Susceptibility mapping in Turkey, Ph.D. Thesis. Department of Geomatics the University of Melbourne, $423 \mathrm{pp}$.

45. Yilmaz, C., T. Topal, and M.L. Suzen. 2012. GIS-based landslide susceptibility mapping using bivariate statistical analysis in Devrek (Zonguldak-Turkey). Environmental Earth Science, 65: 21612178.

46. Zhu, C. and X. Wang. 2009. Landslide susceptibility mapping: A comparison of information and weights-of evidence methods in Three Gorges Area. International Conference on Environmental Science and Information Application Technology, 187: 342-346. 


\title{
A Comparative Assessment between Weights-of-Evidence and Frequency Ratio Models for Landslide Hazard Zonation in Vanak Basin
}

\author{
Ali Reza Arabameri ${ }^{1}$, Kuorosh Shirani ${ }^{2}$ and Khalil Rezaeei ${ }^{3}$ \\ 1- PhD Student of Geomorphology, Faculty of Geomorphology, Tarbiat Modarres University and Teacher of the \\ Faculty of Earth Sciences, Damghan University \\ (Corresponding Author: Alireza.ameri91@ yahoo.com) \\ 2- Assistant professor, Isfahan Research Center for Agriculture and Natural Resources, Isfahan, Iran \\ 3- Assistant professor, Department of Geology, Faculty of Earth Sciences, Kharazmi University \\ Received: February 2, $2016 \quad$ Accepted: June 19, 2016
}

\begin{abstract}
In this study, Vanak catchment because of high sensitivity to landslide was selected. Then with geological, topographic maps and field survey, Landslide hazard map was prepared using GPS as dependent variables. A total of 110 landslides were mapped in GIS out of which 77 (70\%) locations were chosen for the modeling purpose and the remaining $33(30 \%)$ points were used for the model validation. Then layers of the landslide conditioning factors including slope degree, slope aspect, plan curvature, altitude, lithology, land use, distance of road, distance of fault, distance of drainage, drainage density, topographic wetness index (TWI) and Normalized Difference Vegetation Index (NDVI) Calculated. The relationship between the predisposing factors and the landslides were calculated using weights-of-evidence and Frequency Ratio Models. Finally, the susceptibility map was classified into five susceptibility classes: very low, low, moderate, high, and very high. In order to verification, the results were compared with landslides which were not used during the training of the models. Subsequently, the Receiver Operating Characteristic (ROC) curves were drawn and the area under curves (AUC) were calculated for landslide susceptibility maps. Results obtained from validation showed that AUC for Frequency Ratio and weights-of-evidence models are 0.917 (91.7\%) and $0.890(89.0 \%)$, Therefore, the results revealed that the Frequency Ratio model is more suitable than the weights-of-evidence model. Finally, verification indicates satisfactory agreement between resulted susceptibility map and existing data on landslide location.
\end{abstract}

Keywords: Frequency Ratio model, Landslide, Vanak Basin, Weights-of-evidence, Zonation model 